\title{
On mapping models of field lines in a stochastic magnetic field
}

\author{
S.S. Abdullaev \\ Institut für Plasmaphysik, Forschungszentrum Jülich GmbH, EURATOM Association, \\ Trilateral Euregio Cluster, D-52425 Jülich, Germany \\ Received 10 November 2003, accepted for publication 15 March 2004 \\ Published 11 May 2004 \\ Online at stacks.iop.org/NF/44/S12 \\ DOI: $10.1088 / 0029-5515 / 44 / 6 / \mathrm{S} 02$
}

\begin{abstract}
Mapping models of Hamiltonian systems are discussed using the example of magnetic field lines in magnetically confined fusion plasmas. They are usually constructed in a certain symplectic form by imposing several constraints that make them compatible with a toroidal geometry. The possible symplectic forms of model mappings for Hamiltonian systems are derived using the recently developed method for the construction of symplectic mappings (Abdullaev S.S. 2002 J. Phys. A 35 2811). It is shown that the symplectic mappings may have symmetric and nonsymmetric forms. The generating function of the symmetric mapping depends only on the perturbation field while the one for the nonsymmetric map depends also on the safety factor (or winding number). Mapping models, particularly, a global mapping model in a toroidal system, the tokamap (Balescu R. et al 1998 Phys. Rev. E 58 951), usually used in plasma physics are reviewed and their relations with continuous Hamiltonian systems are discussed. The symmetric form of the tokamap is proposed and compared with the conventional tokamap. The symmetric and nonsymmetric mapping models for field lines in ergodic divertor tokamaks are also considered. It is shown that symmetric mappings closely describe original Hamiltonian systems in comparison with nonsymmetric mappings.
\end{abstract}

PACS numbers: 52.55.Fa, 05.45.+b, 52.25.Gj, 05.40.+j

\section{Introduction}

Discrete iterative mappings are powerful tools to study the dynamics of Hamiltonian systems, particularly field lines in magnetically confined toroidal plasmas. Poincaré surface to surface maps simplify the study of three-dimensional magnetic field lines by replacing them with two-dimensional maps, and visualize the dynamics of the system at certain sections (Poincaré sections) [1]. Many concepts of continuous systems become clearer when they are formulated using Poincaré maps. For instance, the study of stability of periodic orbits simply reduces to a study of stability of periodic points of mappings. From the computational point of view they are much faster than a numerical integration of the equations of motion.

The important feature of magnetic field lines consists of the fact that they are described by the one-and-half degree-offreedom Hamiltonian system (see, e.g. [2-4]). The magnetic flux-preserving property of field lines is manifested in the fundamental property of Hamiltonian systems, namely, in the conservation of certain invariants in phase space. This property is known as symplecticness, and the geometry of Hamiltonian systems is called symplectic geometry ${ }^{1}$ [5].

\footnotetext{
1 The word 'symplectic' comes from Greek meaning 'twining or plaiting together', since the canonical coordinate and canonical momentum are intertwined by the symplectic 2 -form.
}

In a numerical study of Hamiltonian systems, it is important to preserve this property. Ordinary numerical methods (like Runge-Kutta methods) are not ideal for integrating Hamiltonian systems because the numerical approximations introduce non-Hamiltonian perturbations that lead to completely different long-term behaviour. Since the structures of magnetic fields in tokamaks and stellarators, like magnetic islands, flux surfaces, ergodic zones, etc are determined by the long-term behaviour of field lines, it is important to use methods preserving an invariant of the Hamiltonian system which is equivalent to the magnetic field flux. Symplectic mappings have a fundamental importance for this purpose.

In plasma physics a mapping approach has been widely used to model the wave-particle interactions and the global behaviour of magnetic field lines in fusion plasmas (see [6-12]). Besides, these mappings are used to study the specific problems of magnetic confinement devices, for instance, the field lines in the presence of so-called ergodic divertors (see [13-19]) and in poloidal divertor tokamaks [20-28].

Mappings are also extensively used in accelerator physics to study the stability of single-particle motion in accelerator devices [29-32]. In dynamical astronomy different symplectic mapping methods have been proposed to simulate the 
long-term evolution of planetary systems in the Solar System (see [33-38] and references therein).

Furthermore, following [37] I will call these mappings mapping models of Hamiltonian systems since they are not a substitute for direct integration of the equations of motion. In majority of cases they have not been rigorously obtained from continuous Hamiltonian equations of motion. The main goal of mapping models is to replace the Poincaré maps of original dynamical systems by iterative maps. What is most important, however, is that constructed in a symplectic form these maps conserve invariants of Hamiltonian systems. Moreover, the symplectic maps run much faster than small-step numerical integration methods, which usually require long computational times to achieve a sufficient accuracy.

The most difficult task in this problem, which is also an important one, is to construct a mapping model that adequately replaces the continuous equations of motion. Mapping models should retain and reproduce the main properties of the original Hamiltonian system. At the same time one wishes to have equations for mappings that are as simple as possible. These mapping models constructed ab initio should be checked for their relevance to continuous system a posteriori [11].

There are several constraints which are usually imposed on mapping models. The first important requirement is that the map should be Hamiltonian (or symplectic), i.e. it should retain the flux-preserving property of the magnetic field. Usually, this requirement is satisfied by choosing the special symplectic form of mappings. Other important constraints are that the model mapping should have the same (periodic) fixed points as the Poincaré map of the original system with the correct stability, and it should have the same regular and chaotic regions as the original system [37]. In the case of systems where perturbations are not exactly known one imposes other requirements that originate from some specific properties of the system. For instance, in toroidal magnetic confinement devices field lines on the magnetic axis should be mapped to themselves, and the magnetic flux should always be positive [11].

There are several shortcomings in these mapping models. The main shortcoming lies in the fact that one often chooses one particular symplectic form of mappings which does not represent the original system uniquely. There exist other forms of mappings which may equivalently represent systems. In general, the different symplectic forms yield different results. Since, mappings are not rigorously derived from continuous Hamiltonian systems, in most cases, it is not clear which form of mappings represents the original system more correctly.

In this work, we shall discuss the mapping models of magnetic field lines in magnetically confined plasmas based on rigorous methods to construct symplectic mappings for Hamiltonian systems that were recently developed in $[39,40]$. This analysis can also be used in other areas of physics and dynamical astronomy where symplectic mapping models have been widely employed to model different physical processes.

The work consists of six sections. In section 2 , we recall the Hamiltonian formulation of equations of magnetic field lines and the mapping models of field lines. Methods of derivation of symplectic mappings from continuous Hamiltonian systems are discussed in section 3. First, we describe conventional methods to construct symplectic mappings, their difficulties and shortcomings. Then, we derive possible forms of symplectic mappings using the rigorous perturbative method to construct mappings for Hamiltonian systems. In section 4, the relation between the mapping model describing the global dynamics of field lines in magnetically confined plasma, a tokamap, proposed by Balescu et al [11], and a continuous Hamiltonian system is discussed. Using the results of section 3 the symmetric form of the tokamap is proposed and compared with the tokamap and the corresponding continuous system. In section 5, we consider mapping models to describe magnetic field lines in a tokamak in the presence of external magnetic perturbations. Finally, in section 6 we summarize the main results and make general remarks on mapping models.

\section{Hamiltonian field line equations and mappings}

Magnetic field lines are three-dimensional curves, $\boldsymbol{x}(s)=$ $(x(s), y(s), z(s))$, tangent to a magnetic field $\boldsymbol{B}$. They are determined by a set of equations,

$$
\frac{\mathrm{d} \boldsymbol{x}}{\mathrm{d} s}=\boldsymbol{B}
$$

where $s$ is an independent parameter related to the element of length along the field line $\mathrm{d} l=\left(\mathrm{d} x^{2}+\mathrm{d} y^{2}+\mathrm{d} z^{2}\right)^{1 / 2}: \mathrm{d} s=$ $|\boldsymbol{B}|^{-1} \mathrm{~d} l$. In magnetically confined plasmas, like tokamaks and stellarators, magnetic field lines lie on nested toroidal surfaces, magnetic surfaces, wound around a circular closed magnetic field line, the magnetic axis. The magnetic surfaces are labelled by a so-called toroidal flux, $\psi$, equal to a magnetic flux through the surface perpendicular to the magnetic axis where $\psi=0$. The position of the field line coordinate on the magnetic surface is uniquely given by a poloidal angle, $\vartheta$ (the short way around the torus) and toroidal angle, $\varphi$ (the long way around the torus). Below, we use the magnetic flux $\psi$ normalized to its value at the plasma boundary. Therefore, $\psi=1$ stands for the plasma boundary. For the plasma with a circular cross section we have $\psi=r^{2} / a^{2}$, where $r$ is the radial coordinate with respect to the magnetic axis $r=0$, and $a$ is a minor radius of the plasma.

In terms of these variables a divergence-free magnetic field $\boldsymbol{B}$ can always be written in the Clebsch form (see, e.g. $[2,3,41])$

$$
\boldsymbol{B}=\nabla \psi \times \nabla \vartheta+\nabla \varphi \times \nabla H
$$

and the equations for magnetic field lines take the Hamiltonian form:

$$
\frac{\mathrm{d} \psi}{\mathrm{d} \varphi}=-\frac{\partial H}{\partial \vartheta}, \quad \frac{\mathrm{d} \vartheta}{\mathrm{d} \varphi}=\frac{\partial H}{\partial \psi},
$$

with $(\vartheta, \psi)$ the canonical variables, $\varphi$ the independent timelike variable, and the function $H=H(\vartheta, \psi, \varphi)$, a poloidal flux, playing the role of a Hamiltonian. It is a $2 \pi$-periodic function of $\vartheta$ and $\varphi$. The formulation of magnetic field line equations in these variables corresponds to the formulation of Hamiltonian equations in action-angle variables $(I, \vartheta)$ [5].

The unperturbed case corresponds to the equilibrium magnetic field configuration with the nested magnetic surfaces $\psi(x, y, z)=$ const. The Hamiltonian $H$ in this case depends 
only on the toroidal flux $\psi$, i.e. $H=H(\psi)$, and the field line equations (3) become completely integrable with the following solutions:

$$
\psi=\text { const, } \quad \vartheta=\omega(\psi)\left(\varphi-\varphi_{0}\right),
$$

where

$$
\omega(\psi)=\frac{\partial H_{0}(\psi)}{\partial \psi}=\frac{1}{q(\psi)}
$$

is the frequency (or winding number). Its inverse $1 / \omega(\psi)=$ $q(\psi)$ is known as the safety factor. The latter has an interpretation as the number of turns along the toroidal angle $\varphi$ per turn along the poloidal angle $\vartheta$.

The magnetic perturbations, in general, are not uniform along the toroidal, $\varphi$, and poloidal, $\theta$, axes and they break the symmetry of the equilibrium field along the toroidal axis. In the presence of these non-axisymmetric magnetic perturbations the poloidal flux $H$ can be represented as a sum of the unperturbed flux $H_{0}(\psi)$ and the perturbed part of the flux $\epsilon H_{1}=\epsilon H_{1}(\psi, \vartheta, \varphi)$ depending on the poloidal and toroidal angles:

$$
H=H_{0}(\psi)+\epsilon H_{1}(\psi, \vartheta, \varphi), \quad H_{0}(\psi)=\int \frac{\mathrm{d} \psi}{q(\psi)} .
$$

The dimensionless perturbation parameter $\epsilon$ introduced in (5) stands for the relative strength of the magnetic perturbations. Since the perturbed part of the Hamiltonian (or the perturbation Hamiltonian) $H_{1}$ is a $2 \pi$-periodic function of $\vartheta$ and $\varphi$, it can be always written as a Fourier series:

$$
H_{1}(\psi, \vartheta, \varphi)=\sum_{m, n} H_{m n}(\psi) \cos \left(m \vartheta-n \varphi+\chi_{m n}\right)
$$

The integer numbers $m$ and $n$ are called the poloidal and toroidal mode numbers, respectively, and the constants $\chi_{m n}$ represent their phases.

\subsection{Mapping of field lines}

As was mentioned above integration of the field line equation (3) with the Hamiltonians (5) and (6) using the standard numerical methods, like Runge-Kutta, is not ideal for this purpose because they are not symplectic, i.e. they do not preserve the flux-conservation property of the magnetic field. For this reason one should use the symplectic numerical integration schemes developed to integrate Hamiltonian systems (see reviews $[42,43]$ ). However, explicit symplectic integration schemes are not applicable to the Hamiltonian (5) since the perturbation Hamiltonian $H_{1}(\psi, \vartheta, \varphi)$ depends on the toroidal flux $\psi$ and the full Hamiltonian $H$ cannot be written as a sum, $H=T(\psi)+V(\vartheta, \varphi)$, of kinetic, $T(\psi)$, and potential, $V(\vartheta, \varphi)$, parts depending on only one of the variables $(\psi, \vartheta)$, respectively. Implicit symplectic numerical integration schemes applicable to the Hamiltonian system (5) are usually time consuming. Moreover, since the Hamiltonian system of field lines (5) is non-autonomous, i.e. the Hamiltonian $H(\psi, \vartheta, \varphi)$ is a function of canonical 'time' $\varphi$, the methods of symplectic integration employed for autonomous systems may not be valid in the case of field lines.

In the mapping approach to integrate the Hamiltonian system (5) and (6), one wishes to avoid small time-step

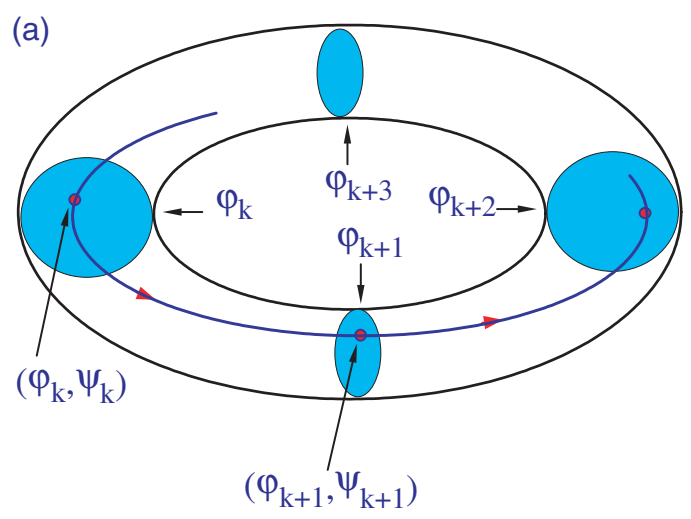

(b)

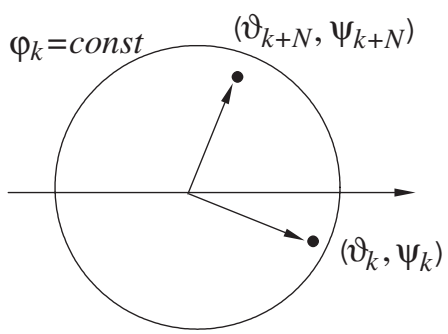

Figure 1. Geometry of mapping in a toroidal system: (a) scheme of the mapping along the toroidal angle; $(b)$ Poincaré return map of field lines on the poloidal plane, $\varphi_{k}=$ const.

integration replacing it by return mappings of the variables $(\vartheta, \psi)$ to a certain poloidal section of the torus $\varphi=\varphi_{k}$. Below, we define a mapping of field lines in a toroidal system. To be more general, we consider the case when a toroidal mode number $n$ takes values proportional to a certain number $N(N \geqslant 1)$, which corresponds to the $N$-fold symmetry of magnetic perturbation along the toroidal angle $\varphi$, i.e. $n=s N$, $(s= \pm 1, \pm 2, \ldots)$. Let $\left(\vartheta_{k}, \psi_{k}\right)$ be the poloidal angle and toroidal flux at the poloidal sections $\varphi=\varphi_{k}=2 \pi \mathrm{k} / \mathrm{N}$, $(k=0, \pm 1, \pm 2, \ldots)$ as illustrated in figure $1(a)$ for the case $N=4$. We define the map

$$
\left(\vartheta_{k+1}, \psi_{k+1}\right)=\hat{M}\left(\vartheta_{k}, \psi_{k}\right),
$$

which relates the variables $(\vartheta, \psi)$ at the successive sections $\varphi_{k}$ and $\varphi_{k+1}$. Then, the map $(\hat{M})^{N}$ defines the Poincaré return map as shown in figure $1(b)$. The flux-preserving property of the mapping (7) is expressed by

$$
\left|\frac{\partial\left(\vartheta_{k+1}, \psi_{k+1}\right)}{\partial\left(\vartheta_{k}, \psi_{k}\right)}\right|=1 \text {. }
$$

\subsection{Mapping models}

Since the early 1980 s different iterative maps (7) have been introduced to study magnetic field lines in plasmas instead of the time consuming integration of field line equations. Although, in most cases, these maps were not rigorously derived from the equations of field lines they are constructed in the symplectic form:

$$
\begin{gathered}
\psi_{k+1}=\psi_{k}-\epsilon \frac{\partial S\left(\vartheta_{k}, \psi_{k+1}\right)}{\partial \vartheta_{k}}, \\
\vartheta_{k+1}=\vartheta_{k}+\frac{2 \pi}{N q\left(\psi_{k+1}\right)}+\epsilon \frac{\partial S\left(\vartheta_{k}, \psi_{k+1}\right)}{\partial \psi_{k+1}},
\end{gathered}
$$


which conserves the flux-preserving property of magnetic field, i.e. satisfies the condition (8). The mapping (9) is determined by the generating function $S\left(\vartheta_{k}, \psi_{k+1}\right)$ and it can be considered as the canonical transformation of variables $\left(\vartheta_{k}, \psi_{k}\right)$ to new ones $\left(\vartheta_{k+1}, \psi_{k+1}\right)$ given by the generating function,

$$
F\left(\vartheta_{k}, \psi_{k+1}\right)=\vartheta_{k} \psi_{k+1}+\frac{2 \pi}{N} \int q^{-1}\left(\psi_{k+1}\right) \mathrm{d} \psi_{k+1}+\epsilon S\left(\vartheta_{k}, \psi_{k+1}\right) .
$$

Then, the map (9) is given by the relation

$$
\psi_{k}=\frac{\partial F\left(\vartheta_{k}, \psi_{k+1}\right)}{\partial \vartheta_{k}}, \quad \vartheta_{k+1}=\frac{\partial F\left(\vartheta_{k}, \psi_{k+1}\right)}{\partial \psi_{k+1}} .
$$

In the absence of perturbation $(\epsilon=0)$ it determines a simple rotational transform given by the safety factor $q(\psi)$. The part of the generating function $\epsilon S$ takes into account the effect of the perturbation $H_{1}$. The symplectic map (9) is known as the perturbed twist map when the frequency $\omega(\psi)=1 / q(\psi)$ is a monotonic function of $\psi$. Below, we briefly recall some important mapping models.

2.2.1. The standard map and its generalizations. This class of mappings corresponds to the symplectic mapping (9) with the generating function $S$ depending only on the poloidal angle $\vartheta$. The general form of the corresponding generating function for $N=1$ is given by [9]

$$
\epsilon S(\vartheta)=\epsilon \sum_{m} g_{m} \cos \left(m \vartheta+\chi_{m}\right),
$$

where $g_{m}$ and $\chi_{m}$ are the constant amplitudes and phases of perturbation modes. The corresponding map has the following form:

$$
\begin{gathered}
\psi_{k+1}=\psi_{k}-\epsilon \sum_{m} g_{m} \sin \left(m \vartheta_{k}+\chi_{m}\right), \\
\vartheta_{k+1}=\vartheta_{k}+\frac{2 \pi}{q\left(\psi_{k+1}\right)} .
\end{gathered}
$$

The standard map (or the Chirikov-Taylor map [44]) can be obtained from (11) by retaining in the generating function $S$ (10) only one mode $m=1$ with $g_{m}=1$ and $\chi_{m}=0$ and choosing the safety factor $q(\psi)=1 / \psi$ :

$$
\begin{aligned}
& \psi_{k+1}=\psi_{k}-\epsilon \sin \vartheta_{k}, \\
& \vartheta_{k+1}=\vartheta_{k}+2 \pi \psi_{k+1} .
\end{aligned}
$$

This map has been widely used to model a field line stochasticity in tokamaks by many authors (see, e.g. [45-49]). Thanks to its simple form it allowed one to efficiently calculate the diffusion coefficients of field lines and the transport of test particles in a magnetic field with destroyed magnetic surfaces. Recently, the standard map has been used to model the test particle transport in a tokamak caused by the drift-wave turbulence $[50,51]$. The more general maps (11) proposed in [9] have been used in [52-54] to study stochastic magnetic field lines in tokamaks.

2.2.2. The Wobig-Mendonça map. In toroidal systems typical magnetic perturbations are radially dependent, i.e. $H_{1}(\psi, \vartheta, \varphi)$ depends on the toroidal flux $\psi$. In order to take into account this feature of the toroidal magnetic field, the generating function $S$ should be chosen as a function that depends also on the poloidal flux $\psi$. The simplest form of such a map proposed by Wobig [6] and later generalized by Mendonça [9]) has the following form:

$$
\begin{gathered}
\psi_{k+1}=\psi_{k}-\epsilon g\left(\psi_{k+1}\right) \sum_{m} g_{m} \sin m \vartheta_{k}, \\
\vartheta_{k+1}=\vartheta_{k}+\frac{2 \pi}{q\left(\psi_{k+1}\right)}-\epsilon g^{\prime}\left(\psi_{k+1}\right) \sum_{m} g_{m} \cos m \vartheta_{k},
\end{gathered}
$$

which corresponds to the map (9) with the generating function

$$
\begin{gathered}
\epsilon S(\vartheta, \psi)=\epsilon g(\psi) \sum_{m} g_{m} \cos m \vartheta, \\
g(\psi)=1-\exp (-\psi),
\end{gathered}
$$

where $g^{\prime}(\psi) \equiv \mathrm{d} g(\psi) / \mathrm{d} \psi$ and $g_{m}$ are constant coefficients. The case when $g(\psi)=\psi$ and $q(\psi)=1 / \psi$ was considered by Wobig [6]. Near the magnetic axis $(\psi=0)$, when $\psi \ll 1$, the function $g(\psi)$ can be approximated by a linear function $\psi$ and the map (13) is reduced to [6]

$$
\begin{gathered}
\psi_{k+1}=\psi_{k}\left(1+\epsilon \sum_{m} g_{m} \sin m \vartheta_{k}\right)^{-1}, \\
\vartheta_{k+1}=\vartheta_{k}+\frac{2 \pi}{q\left(\psi_{k+1}\right)}-\epsilon \sum_{m} g_{m} \cos m \vartheta_{k},
\end{gathered}
$$

At regions far from the magnetic axis, when $\psi \gg 1$, the map (13) approaches the mappings (11).

As was noted by Balescu et al [11] the toroidal flux $\psi$ in the standard map (12) may take on negative values after some iterations of the maps for an arbitrary value of $\epsilon$. A similar situation occurs in the Wobig map (15) for $\epsilon>1$. This fact is not compatible with the magnetic field line behaviour in a toroidal system where the toroidal flux $\psi \sim r^{2}$ is always positive. On the other hand, the profile of the safety factor $q \sim r^{-2}$ in both the standard map and the Wobig map, does not represent any realistic case in tokamaks.

2.2.3. The tokamap. A specific form of mapping called the tokamap, which is compatible with toroidal geometry, has been recently proposed by Balescu et al $[11,12]$. It describes the global behaviour of magnetic field lines in tokamaks. The tokamap has been constructed as an iterative map in the symplectic form (9) representing a global picture of a tokamak cross section $\varphi_{k}=2 \pi k(\bmod 2 \pi)$. The generating function $S$ is chosen to be compatible with the toroidal geometry, i.e. the canonical momentum $\psi$ (toroidal flux) should always take positive values. For instance, if $\psi_{0}>0$ at the section $k=0$ then $\psi_{k}>0$ for all $k$; and if $\psi_{0}=0$, then $\psi_{k}=0$ for all $k$. It has been shown in [11] that the following map satisfies these constraints:

$$
\begin{gathered}
\psi_{k+1}=\psi_{k}-\epsilon \frac{\psi_{k+1}}{1+\psi_{k+1}} \sin \vartheta_{k}, \\
\vartheta_{k+1}=\vartheta_{k}+\frac{2 \pi}{q\left(\psi_{k+1}\right)}-\frac{\epsilon}{\left(1+\psi_{k+1}\right)^{2}} \cos \vartheta_{k} .
\end{gathered}
$$

The perturbation parameter $\epsilon$ takes values in the interval $0<\epsilon<1$. (We have used the safety factor $q(\psi)$ instead 

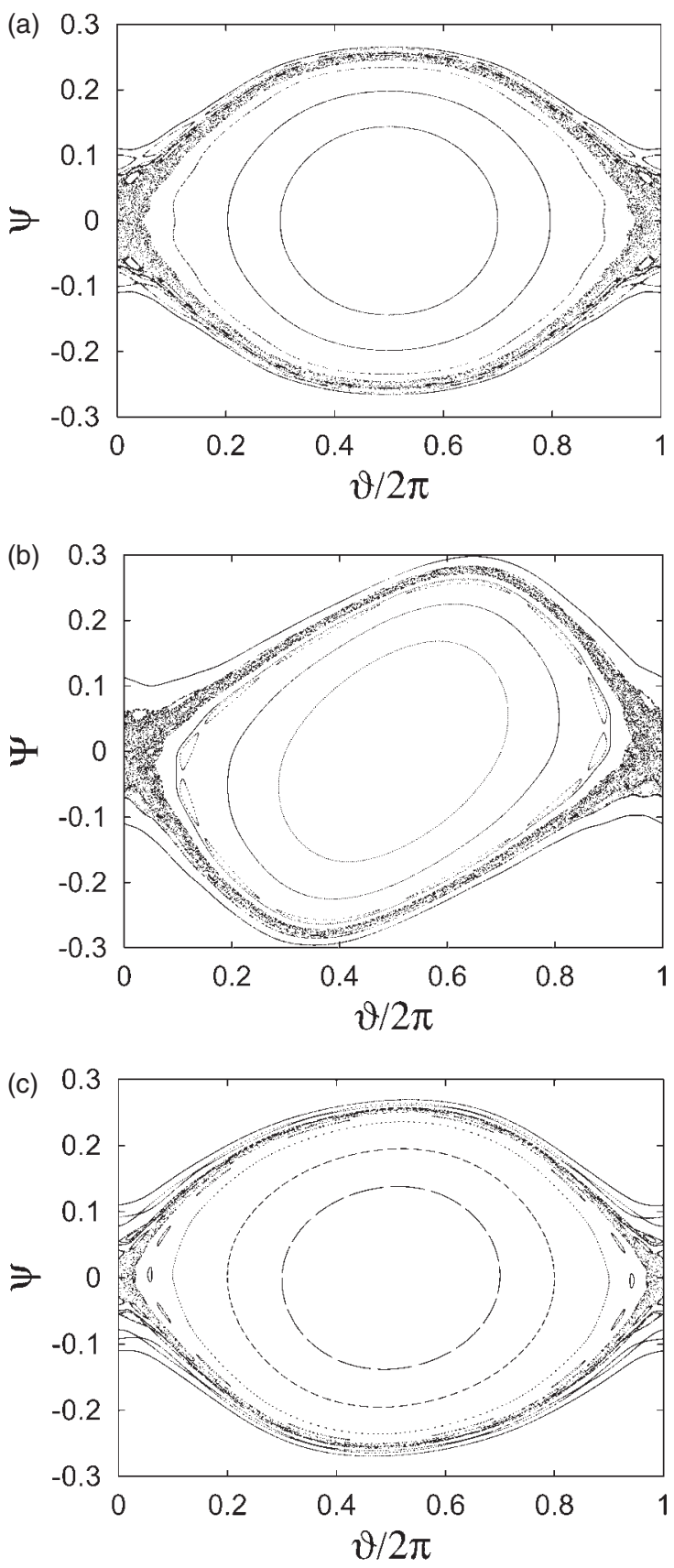

Figure 2. Poincaré sections for the standard Hamiltonian in the $(\vartheta, I)$-plane obtained by $(a)$ the symmetric standard map (47), (b) the standard map (48), (c) the nonsymmetric map (9) with the generating function (45); parameter $\epsilon=0.7 / 2 \pi$.

of the winding number $W(\psi)=1 / q(\psi)$ and the parameter $\epsilon$ instead of the parameter $K$ as in the original paper by Balescu et al [11].) The generating function $S(\vartheta, \psi)$ associated with the tokamap is

$$
\epsilon S(\vartheta, \psi)=-\epsilon \frac{\psi}{1+\psi} \cos \vartheta
$$

and the safety factor $q(\psi)$ can be chosen arbitrary. In [11] the following analytical form of $q(\psi)$ has been used,

$$
q(\psi)=\frac{4 q(0)}{(2-\psi)\left(2-2 \psi+\psi^{2}\right)},
$$
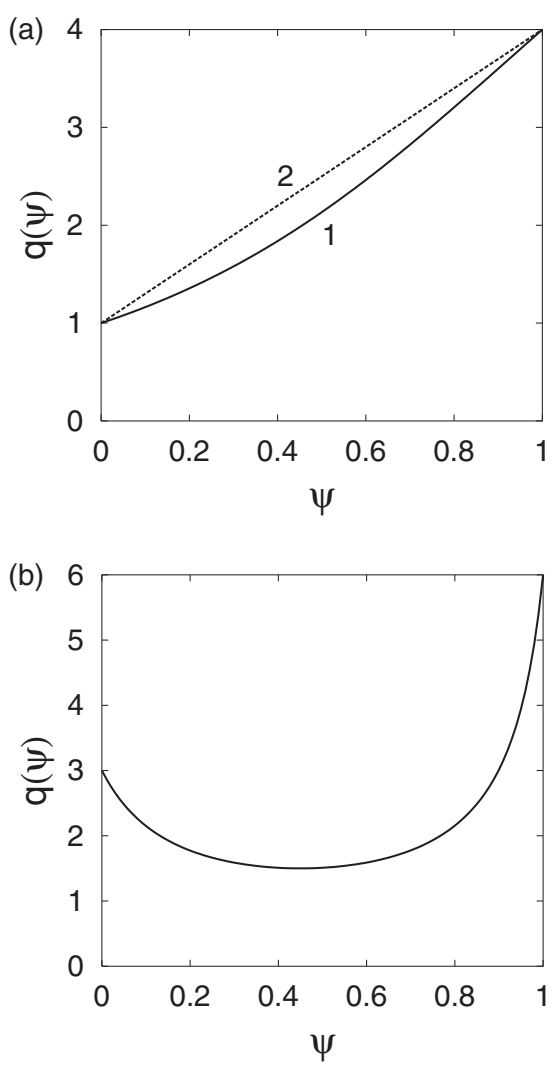

Figure 3. Profiles of the safety factor $q(\psi):(a)$ a monotonic profile (18) (curve 1) and a linear profile $q(\psi)=q_{0}+\left(q_{1}-q_{0}\right) \psi$ (curve 2) at values $q_{0}=1, q_{1}=4 ;(b)$ the non-monotonic profile (19) at the values: $q_{0}=3, q_{m}=1.5$, and $q_{1}=6$.

where $q(0)$ is the value of the safety factor on the magnetic axis $\psi=0$. At the plasma edge $\psi=1$ it takes four times the central value, $q(1)=4 q(0)$. The profile of $q(\psi)(18)$ is shown in figure $3(a)$.

If the profile of $q(\psi)$ is a non-monotonic function of $\psi$ then the tokamap becomes a non-twist map, i.e. a map with a non-monotonic dependence of frequency $\omega(\psi)$ on $\psi$ (see $[55,56,4])$. The corresponding tokamap called revtokamap has been studied by Balescu [12] for the following $q$-profile

$$
q(\psi)=\frac{q_{m}}{1-a\left(\psi-\psi_{m}\right)^{2}},
$$

where $q_{m}$ is a minimum value of $q$ at $\psi=\psi_{m}$. The profile of the safety factor (19) is shown in figure 3(b). Parameters $a$ and $\psi_{m}$ can be written in terms of the values of $q(\psi)$ at the axis $\psi=0, q_{0}=q(0)$, and at the plasma edge $\psi=1, q_{1}=q(1)$, respectively,

$$
\psi_{m}=\left[1+\left(\frac{1-q_{m} / q_{1}}{1-q_{m} / q_{0}}\right)^{1 / 2}\right]^{-1}, \quad a=\frac{1-q_{m} / q_{0}}{\psi_{m}^{2}} .
$$

The revtokamap describes the dynamics of field lines in a tokamak magnetic configuration with a so-called reversed magnetic shear. The latter corresponds to the profile of the safety factor $q(\psi)$ which has a minimum not at the magnetic axis $\psi=0$, but at a normalized radius of $0.3-0.4$ and regularly increases towards the centre $(\psi=0)$ and the edge of the 
plasma $(\psi=1)$. Recent tokamak experiments (see, for instance, reviews $[57,58]$ ) have clearly shown the appearance of improved confinement regimes, internal transport barriers (ITBs), in the presence of a reversed magnetic shear.

The first equation in (16) can be explicitly solved with respect to the variable $\psi_{k+1}$ :

$$
\psi_{k+1}=\frac{1}{2}\left[\sqrt{P^{2}\left(\psi_{k}, \vartheta_{k}\right)+4 \psi_{k}}-P\left(\psi_{k}, \vartheta_{k}\right)\right],
$$

where $P\left(\psi_{k}, \vartheta_{k}\right)=1-\psi_{k}+\epsilon \sin \vartheta_{k}$. The tokamap has been used to model the formation of transport barriers and anomalous subdiffusion of field lines in tokamaks [59-61].

Although the tokamap has not been directly derived from the equations of field lines it has recently been recovered in [62] from the particle map of the guiding centre motion in a toroidal system described by the standard magnetic field in the presence of the magnetic field perturbation. As was stated in [62] it is deduced as a particular case of the particle map in the limit of zero magnetic moment and when only a simple $m=0$ non-resonant magnetic perturbation is applied. The relation between the tokamap and the continuous field line equations has also been studied in [63].

A direct derivation of symplectic mappings, particularly a tokamap, from the field line equations (3), (5) and (6) would allow us to study the relation between mapping models and the corresponding magnetic perturbations. We discuss this problem in section 4.1 .

\section{Methods to construct symplectic mappings}

Until recently there were several widely used methods to derive symplectic mappings. They can be roughly divided into two classes. The first class can be called the method of a priori assumptions, and the second one the method of delta functions. A short description of these methods and their shortcomings will be given below. We also describe rigorous methods to construct mappings recently developed in $[39,40]$ and use this method to obtain the mapping models.

\subsection{Conventional methods}

In the method of a priori assumptions it is assumed that the mapping has a given symplectic form (9) and the generating function $S$ is derived from the equations of motion (see, e.g. $[64,65])$. Supposing that the perturbation is small $(\epsilon \ll 1)$ one obtains

$$
\begin{aligned}
& S\left(\vartheta_{k}, \psi_{k+1}\right)=\int \mathrm{d} \vartheta_{k} \\
& \quad \times\left\{\int_{0}^{2 \pi / N} f\left(\psi_{k+1}, \vartheta_{k}+\omega\left(\psi_{k+1}\right)\left(\varphi-\varphi_{k}\right), \varphi\right) \mathrm{d} \varphi\right\}, \\
& f(\psi, \vartheta, \varphi)=\frac{\partial H_{1}(\psi, \vartheta, \varphi)}{\partial \vartheta},
\end{aligned}
$$

where the integral is taken over unperturbed trajectories $\psi_{0}(\varphi)=\psi_{k+1}, \vartheta_{0}(\varphi)=\omega\left(\psi_{k+1}\right)\left(\varphi-\varphi_{k}\right)+\vartheta_{k}$ over one period, $2 \pi / N$, of the toroidal angle $\varphi$.

A similar approach has been proposed in $[36,37]$ to construct mapping models for autonomous Hamiltonian systems in dynamical astronomy. This method is based on the averaging procedure of the original Hamiltonian system, and constructing the mapping in the form (9) in terms of the slow variables of averaged Hamiltonian system. In our notation the corresponding generating function $S$ of the mapping is equal to $(2 \pi / N) \mathcal{H}_{1}\left(\psi_{k+1}, \vartheta_{k}\right)$, where $\mathcal{H}_{1}\left(\psi_{k+1}, \vartheta_{k}\right)$ is the averaged Hamiltonian and $\left(\vartheta_{k}, \psi_{k}\right)$ are slowly changing variables.

Using a similar procedure one can construct a symplectic map in a slightly different form

$$
\begin{gathered}
\psi_{k+1}=\psi_{k}+\epsilon \frac{\partial S\left(\vartheta_{k+1}, \psi_{k}\right)}{\partial \vartheta_{k+1}}, \\
\vartheta_{k+1}=\vartheta_{k}+\frac{2 \pi}{N q\left(\psi_{k}\right)}-\epsilon \frac{\partial S\left(\vartheta_{k+1}, \psi_{k}\right)}{\partial \psi_{k}},
\end{gathered}
$$

which is an equivalent description of the system as the perturbed map in the form (9). The difference between the two symplectic forms (9) and (22) is that the variable $\psi_{k+1}$ in the map (9) is defined implicitly, while in (22) the angle variable $\vartheta_{k+1}$ is defined implicitly.

However, the method of deriving the symplectic mappings that has been described has significant shortcomings. First of all, it restricts the possible forms of symplectic maps to only two, given by (9) and (22). Second, it does not allow us to obtain the higher order corrections in the perturbation parameter $\epsilon$ and, therefore, to estimate the accuracy of replacing the continuous system (5) by symplectic mappings.

The method of delta functions is often used to obtain symplectic maps for the Hamiltonian system affected by timeperiodic perturbations with a broad spectrum (see, e.g. [9, 33, $44,66,67]$ ). For such a system the perturbation Hamiltonian can be presented in the form

$H_{1}(\psi, \vartheta, t)=\sum_{m} \sum_{s=-M}^{M} H_{m, s N}(\psi) \cos \left(m \vartheta-s N \varphi+\chi_{m, s N}\right)$,

with a finite but large number of harmonics $M \gg 1$. Supposing that in equation (23) the amplitudes, $H_{m n}(I)$, and phases, $\chi_{m n}(I)$, with $H_{m}(I)$, do not depend on the index $n=s N$, i.e. $H_{m n}(I) \equiv H_{m}(I), \chi_{m n}(I) \equiv \chi_{m}(I)$, and extending the mode number $M$ to infinity $(M \rightarrow \infty)$ the perturbation Hamiltonian (23) can be reduced to

$H_{1}(\psi, \vartheta, \varphi)=\mathcal{H}(\psi, \vartheta) \frac{2 \pi}{N} \sum_{k=-\infty}^{\infty} \delta\left(\varphi-\frac{2 \pi}{N} k\right)$.

Then, the equations for the perturbed motion are

$$
\begin{gathered}
\frac{\mathrm{d} \vartheta}{\mathrm{d} \varphi}=\omega(\psi)+\epsilon g(\vartheta, \psi) \sum_{k=-\infty}^{\infty} \delta\left(\varphi-\frac{2 \pi}{N} k\right), \\
\frac{\mathrm{d} \psi}{\mathrm{d} \varphi}=\epsilon f(\vartheta, \psi) \sum_{k=-\infty}^{\infty} \delta\left(\varphi-\frac{2 \pi}{N} k\right),
\end{gathered}
$$

where the perturbation functions

$$
g(\vartheta, \psi)=\frac{\partial S(\vartheta, \psi)}{\partial \psi}, \quad f(\vartheta, \psi)=-\frac{\partial S(\vartheta, \psi)}{\partial \vartheta}
$$

are given by the generating function

$$
S(\vartheta, \psi)=\frac{2 \pi}{N} \sum_{m} H_{m}(\psi) \cos \left(m \vartheta+\chi_{m}\right) .
$$


The justification for the replacement of the continuous perturbation function (23) by a series of delta functions is based on the averaging principle $[33,34]$. It is based on the fact that if high-frequency terms do not contribute significantly to the evolution, then adding these terms also does affect the system significantly. However, one should note that such a replacement of (23) by (24) introduces artificial singularities and discontinuities into the system at the sections $\varphi_{k}=2 \pi k$. The trajectory $\psi(\varphi), \vartheta(\varphi)$ is not defined at these sections.

It is often supposed that integrating the equation (25) over one period from $\varphi_{k}-0$ to $\varphi_{k}-0+2 \pi / N$, one obtains the map (7). In general, the procedure of integration over products of delta function with discontinuous functions $f(\psi, \vartheta)$, $g(\psi, \vartheta)$, i.e.

$$
\int_{\varphi_{k}-0}^{\varphi_{k}+0} f(\vartheta(\varphi), \psi(\varphi)) \delta\left(\varphi-\varphi_{k}\right) \mathrm{d} \varphi
$$

is not well defined. If the integral (27) is replaced by $f\left(\psi_{k+1}, \vartheta_{k}\right)$ we would obtain the map of type (9) with the generating function (26). This assumption is often used in the literature (see, e.g. $[9,14,17-19]$ ) in spite of the fact that this procedure is not completely justified.

Difficulties in obtaining symplectic maps using this method have also been discussed in $[37,38,63]$. Depending on the definition of the delta function one can obtain different forms of mappings. However, as was shown in [37,38] they may not be symplectic. Particularly, in [63] it has been found that the integration of the system (25) from $\varphi_{k}$ to $\varphi_{k+1}$ using the symmetric definition of the $\delta$-function,

$$
\int_{2 \pi k}^{2 \pi k \pm 0} f(\psi, \vartheta) \delta(\varphi-2 \pi k) \mathrm{d} \varphi= \pm \frac{1}{2} f\left(\psi_{k}, \vartheta_{k}\right),
$$

where $\left(\psi_{k}, \vartheta_{k}\right)=\left(\psi\left(\varphi_{k}\right), \vartheta\left(\varphi_{k}\right)\right)$, gives rise to the following symmetric form of the map (in our notations)

$$
\begin{gathered}
\psi_{k}^{+}=\psi_{k}-\frac{\epsilon}{2} \frac{\partial S\left(\vartheta_{k}, \psi_{k}\right)}{\partial \vartheta_{k}}, \\
\vartheta_{k}^{+}=\vartheta_{k}+\frac{\epsilon}{2} \frac{\partial S\left(\vartheta_{k}, \psi_{k}\right)}{\partial \psi_{k}}, \\
\vartheta_{k+1}^{-}=\vartheta_{k}^{+}+\frac{2 \pi}{N q\left(\psi_{k}^{+}\right)}, \quad \psi_{k+1}^{-}=\psi_{k}^{+}, \\
\psi_{k+1}=\psi_{k+1}^{-}-\frac{\epsilon}{2} \frac{\partial S\left(\vartheta_{k+1}, \psi_{k+1}\right)}{\partial \vartheta_{k+1}}, \\
\vartheta_{k+1}=\vartheta_{k+1}^{-}+\frac{\epsilon}{2} \frac{\partial S\left(\vartheta_{k+1}, \psi_{k+1}\right)}{\partial \psi_{k+1}},
\end{gathered}
$$

The numerical integration of the continuous system (25) by replacing the $\delta$-function by its continuous representation $\delta(t)=\exp \left(-t^{2} / a^{2}\right) / a \sqrt{\pi},(a \ll 1)$ shows good agreement with the map (28) rather than with the symplectic map (9). Particularly, it has been shown that the difference between the tokamap and the corresponding continuous Hamiltonian system is relatively large [63]. However, the symmetric map (28) constructed is also not symplectic, i.e. $J=$ $\left|\partial\left(\psi_{k+1}, \vartheta_{k+1}\right) / \partial\left(\psi_{k}, \vartheta_{k}\right)\right| \neq 1$.

Only in the case when the function $\mathcal{H}_{1}$ does not depend on the action variable $\psi$, i.e. $\partial \mathcal{H}_{1} / \partial \psi=g(\psi, \vartheta) \equiv 0$, does the angle $\vartheta$ become continuous along $\varphi$, and the integration gives rise to the map

$$
\begin{gathered}
\vartheta_{k+1}=\vartheta_{k}+2 \pi \omega\left(\psi_{k+1}\right), \\
\psi_{k+1}=\psi_{k}+\epsilon f\left(\vartheta_{k}\right),
\end{gathered}
$$

which is known as a radial twist map when the frequency $\omega(\psi)$ is a monotonic function of $\psi$.

One should note that in the map (29) the variable $\psi_{k}$ is taken as $\psi_{k}=\psi\left(\psi_{k}-0\right)$. This should be kept in mind when one compares the original continuous system with the perturbation (23), with the approximated one (24). The trajectories $\vartheta(\varphi), \psi(\varphi)$ of the original system are continuous functions of $\varphi$, while its replacement by (24) introduces discontinuities at the sections $\varphi=\varphi_{k}$ where the variables $\left(\vartheta_{k}, \psi_{k}\right)$ in the map (7) should be defined. However, for the system (24) they are not defined at these sections. Therefore, the variables of the map (29) are not identical to those of the original system. The approach that we have presented to construct symplectic maps does not allow us to find the relation between the variables of the original system and the ones in the map (29).

The fact that the variables in the original equations (3) and the ones in the mappings are not identical was also noticed in [35]. In order to relate these variables, so-called symplectic correctors were introduced by means of a Lie formalism.

\subsection{The Hamilton-Jacobi method to construct mappings}

A symplectic mapping method for integrating the Hamiltonian system (3), (5) and (6) based on the Hamilton-Jacobi method and perturbation theory has been developed in $[39,40]$. The general form of the mapping (7) for the Hamiltonian system (5) and (6) is given by the following symmetric flux-preserving form [40]:

$$
\begin{gathered}
\Psi_{k}=\psi_{k}-\epsilon \frac{\partial S^{(k)}}{\partial \vartheta_{k}}, \quad \Theta_{k}=\vartheta_{k}+\epsilon \frac{\partial S^{(k)}}{\partial \Psi_{k}}, \\
\Psi_{k+1}=\Psi_{k}, \quad \bar{\Theta}_{k}=\Theta_{k}+w\left(\Psi_{k}, \epsilon\right)\left(\varphi_{k+1}-\varphi_{k}\right), \\
\psi_{k+1}=\Psi_{k+1}+\epsilon \frac{\partial S^{(k+1)}}{\partial \vartheta_{k+1}}, \quad \vartheta_{k+1}=\bar{\Theta}_{k}-\epsilon \frac{\partial S^{(k+1)}}{\partial \Psi_{k+1}},
\end{gathered}
$$

where $w(\Psi, \epsilon)=\partial \mathcal{H} / \partial \Psi$ is the frequency of perturbed motion, and $S^{(k)} \equiv S\left(\vartheta_{k}, \Psi_{k}\right)$ is the value of the generating function $G\left(\vartheta, \Psi, \varphi, \varphi_{0} ; \epsilon\right)$ taken at sections $\varphi=\varphi_{k}$, i.e. $S\left(\vartheta_{k}, \Psi_{k}\right)=G\left(\vartheta_{k}, \Psi_{k}, \varphi_{k}, \varphi_{0} ; \epsilon\right)$. The generating function obeys the Hamilton-Jacobi equation,

$$
H\left(\Psi+\epsilon \frac{\partial G}{\partial \vartheta}, \vartheta, \varphi\right)+\epsilon \frac{\partial G}{\partial \varphi}=\mathcal{H}(\Psi, \epsilon)
$$

in the finite interval $\varphi_{k}<\varphi<\varphi_{k+1}$ and satisfies the condition $\left.G\right|_{\varphi=\varphi_{0}}=0$ at the initial value $\varphi_{0}\left(\varphi_{k}<\varphi_{0}<\varphi_{k+1}\right)$. The solution of equation (31) with this initial condition is found using the expansion of equation (31) in a perturbation series: the new Hamiltonian, $\mathcal{H}(\Psi, \epsilon)$ and the generating function are represented as a power series expansions in terms of the small perturbation parameter $\epsilon$ :

$$
\mathcal{H}(\psi, \epsilon)=H_{0}(\psi)+\epsilon \mathcal{H}_{1}(\psi)+\epsilon^{2} \mathcal{H}_{2}(\psi)+\cdots,
$$

$G\left(\vartheta, \Psi, \varphi, \varphi_{0} ; \epsilon\right)=G_{1}\left(\vartheta, \Psi, \varphi, \varphi_{0}\right)+\epsilon G_{2}\left(\vartheta, \Psi, \varphi, \varphi_{0}\right)+\cdots$, 
The equations for the expansion coefficients $G_{i} \equiv$ $G_{i}\left(\vartheta, \psi, \varphi, \varphi_{0}\right)$ are

$\frac{\partial G_{1}}{\partial \varphi}+\frac{\partial H_{0}}{\partial \Psi} \cdot \frac{\partial G_{1}}{\partial \vartheta}=-H_{1}(\Psi, \vartheta, \varphi)$,

$\frac{\partial G_{j}}{\partial \varphi}+\frac{\partial H_{0}}{\partial \Psi_{i}} \cdot \frac{\partial G_{j}}{\partial \vartheta}=-F_{j}\left(\Psi, \vartheta, \varphi, \varphi_{0}\right), \quad j \geqslant 2$.

where $F_{j}\left(\Psi, \vartheta, \varphi, \varphi_{0}\right)$ is the polynomial function of derivatives $\partial G_{1} / \partial \vartheta, \ldots, \partial G_{j-1} / \partial \vartheta$. Particularly, for $F_{2}\left(\Psi, \vartheta, \varphi, \varphi_{0}\right)$ we have

$F_{2}\left(\Psi, \vartheta, \varphi, \varphi_{0}\right)=\frac{\partial^{2} H_{0}}{\partial \Psi^{2}} \cdot\left(\frac{\partial G_{1}}{\partial \vartheta}\right)^{2}+\frac{\partial H_{1}}{\partial \Psi} \frac{\partial G_{1}}{\partial \vartheta}$.

The first-order generating function $G_{1}\left(\vartheta, \Psi, \varphi, \varphi_{0}\right)$ in the finite interval $\varphi_{k+1}<\varphi<\varphi_{k}$ is given by [40]:

$$
\begin{aligned}
G_{1} & \left.\vartheta, \Psi, \varphi, \varphi_{0}\right)=-\left(\varphi-\varphi_{0}\right) \sum_{m, n} H_{m n}(\Psi) \\
& \times\left[a\left(x_{m n}\right) \sin \left(m \vartheta-n \varphi+\chi_{m n}\right)\right. \\
& \left.+b\left(x_{m n}\right) \cos \left(m \vartheta-n \varphi+\chi_{m n}\right)\right],
\end{aligned}
$$

where

$$
\begin{gathered}
a(x)=\frac{1-\cos x}{x}, \quad b(x)=\frac{\sin x}{x}, \\
x_{m n}=\left(\frac{m}{q(\Psi)}-n\right)\left(\varphi-\varphi_{0}\right) .
\end{gathered}
$$

Near resonant magnetic surfaces $\psi_{m n},\left(q\left(\psi_{m n}\right)=m / n\right)$, the actual expansion parameter in equation (33) is $\mu=\epsilon\left(\varphi-\varphi_{0}\right)^{v}$ where $v>1$ and the free parameter $\varphi_{0}$ lies in the interval $\varphi_{k} \leqslant \varphi_{0} \leqslant \varphi_{k+1}$ (see [40]).

The mapping (30) can also be considered as an alternative method of symplectic integration of Hamiltonian systems (5) and (6). As was shown in [40] this map with large integration steps, $\Delta \varphi=\varphi_{k+1}-\varphi_{k}$, comparable with the characteristic periods of the system (e.g. a perturbation period) has the same accuracy as the conventional symplectic integrators (see, e.g. the review [42]) with integration steps two or three orders smaller. The mapping can be applied to Hamiltonian systems with moderately large perturbation $\epsilon \sim 1$ by taking the mapping step $\Delta \varphi=\varphi_{k+1}-\varphi_{k}$ to be sufficiently small.

The mapping (30) is called symmetric if the free parameter is taken exactly in the middle of the interval $\left[\varphi_{k}, \varphi_{k+1}\right]$, i.e. $\varphi_{0}=\left(\varphi_{k}+\varphi_{k+1}\right) / 2$. The nonsymmetric forms of mappings can be obtained by appropriately choosing the parameter $\varphi_{0}$. Particularly, taking $\varphi_{0}=\varphi_{k+1}$ and $\Delta \varphi=\varphi_{k+1}-\varphi_{k}=2 \pi / N$ we obtain the Poincaré return map in the form (9), where $S\left(\vartheta_{k}, \psi_{k+1}\right) \equiv G\left(\vartheta_{k}, \psi_{k+1}, \varphi_{k}, \varphi_{k}+2 \pi / N, \epsilon\right)$. According to (36), to first order in $\epsilon$ the generating function $S\left(\vartheta_{k}, \psi_{k+1}\right)$ of the corresponding mapping is given by

$$
\begin{aligned}
& S(\vartheta, \psi)=\frac{2 \pi}{N} \sum_{m, n} H_{m n}(\psi) \\
& \times\left[a\left(x_{m n}\right) \sin \left(m \vartheta+\chi_{m n}^{\prime}\right)+b\left(x_{m n}\right) \cos \left(m \vartheta+\chi_{m n}^{\prime}\right)\right],
\end{aligned}
$$

where $x_{m n}=2 \pi(m / q(\psi)-n)$ and $\chi_{m n}^{\prime}=\chi_{m n}-n \varphi_{k}$.

Similarly, taking $\varphi_{0}=\varphi_{k}$ we obtain the mapping (22). In practical calculations one truncates the expansion series (33) of the generating function. Then, the accuracy of a mapping is determined by the relative magnitude of neglected terms. In the case of the symmetric map the neglected terms near the resonances are of the order of $R_{m} \sim$ $\left(\epsilon(\Delta \varphi / 2)^{v}\right)^{M+1}$ while for the nonsymmetric maps we have $R_{m} \sim\left(\epsilon(\Delta \varphi)^{\nu}\right)^{M+1}$, where $M$ is the number of retained terms in the expansion (33). Therefore, symmetric maps are more accurate than nonsymmetric maps (see [40] for details).

\subsection{Mapping with a numerical interpolation of the Hamiltonian}

The mapping method requires a knowledge of the safety factor $q(\psi)$ and the perturbation Hamiltonian $H_{1}(\psi, \vartheta, \varphi)$ or its Fourier coefficients $H_{m n}(\psi)$. Determination of these functions analytically for realistic magnetic configurations is somewhat difficult. In order to apply the mapping procedure in these cases one can calculate these functions by the numerical integration of the equations of field lines (1) on a grid of magnetic flux $\psi: \psi_{i}=i \Delta \psi,\left(i=1, \ldots, N_{\psi}\right)$. The functions $q(\psi)$, $H_{m n}(\psi)$ for the arbitrary values of $\psi$ can be interpolated by the cubic splines using their pre-calculated values $q\left(\psi_{i}\right)$, $H_{m n}\left(\psi_{i}\right)$.

In order to display the orbits in real space coordinates $\boldsymbol{x}$ the relations of these coordinates with the variables $(\psi, \vartheta)$ can be found during the numerical integration of the equations of field lines in the absence of magnetic perturbations. For instance, the relation between the toroidal coordinates $(r, \theta, \varphi)$ ( $r$ is a minor radius, $\theta$ a geometrical poloidal angle, different from $\vartheta)$ and $(\psi, \vartheta)$ can be sought in the form of a Fourier series

$$
\begin{gathered}
r=r_{0}+\sum_{m}\left(r_{m}^{(s)}(\psi) \sin m \vartheta+r_{m}^{(c)}(\psi) \cos m \vartheta\right), \\
\theta=\vartheta+\sum_{m} \alpha_{m}(\psi) \sin m \vartheta .
\end{gathered}
$$

The Fourier coefficients $r_{m}^{(s)}(\psi), r_{m}^{(s)}(\psi)$, and $\alpha_{m}(\psi)$ can also be calculated numerically for the same grid coordinates of $\psi$, and their values for arbitrary $\psi$ can be interpolated by the cubic splines.

The procedure described replaces the equations of field lines (1) by the Hamiltonian ones (3), (5) and (6), which allows us to integrate them using the symplectic mapping instead of integrating the equations of field lines (1) using the standard, usually, nonsymplectic numerical integration schemes.

\subsection{Mappings for systems with a broad perturbation spectrum}

In this section we construct mappings for Hamiltonian systems affected by perturbation with a broad spectrum, given by equation (23). As we have seen in section 3.1 the method of delta functions for constructing mappings for these systems encounters difficulties related to the uncertain integration procedure along delta functions. The mathematically correct and physically reasonable approach to construct symplectic maps for these systems would consist of two steps: first, obtaining the map for the finite mode number $M$ and then considering the limit $M \rightarrow \infty$. Such a method would avoid the difficulties mentioned above. 
Using equations (36) and (5), (23) the first order generating function $G_{1}$ can be written as

$$
\begin{gathered}
G_{1}\left(\vartheta, \Psi, \varphi, \varphi_{0}\right)=\operatorname{Re} \mathrm{i} \sum_{m} \mathcal{H}_{m}(\Psi) \mathrm{e}^{\mathrm{i} m \vartheta} \\
\times \sum_{n=-M}^{M} \frac{\exp (-\mathrm{i} n N \varphi)}{m \omega(\Psi)-n N}\left(1-\mathrm{e}^{-\mathrm{i}(m \omega(\Psi)-n N)\left(\varphi-\varphi_{0}\right)}\right) \\
=\operatorname{Re} \mathrm{i} \sum_{m} \mathcal{H}_{m}(\Psi) \mathrm{e}^{\mathrm{i} m \vartheta}\left(\sum_{n=-M}^{M} \frac{\exp (-\mathrm{i} n N \varphi)}{m \omega(\Psi)-n N}\right. \\
\left.\quad-\mathrm{e}^{-\mathrm{i} m \omega(\Psi)\left(\varphi-\varphi_{0}\right)} \sum_{n=-M}^{M} \frac{\exp \left(-\mathrm{i} n N \varphi_{0}\right)}{m \omega(\Psi)-n N}\right)
\end{gathered}
$$

where $\mathcal{H}_{m}(\psi)=H_{m}(\psi) \exp \left(\mathrm{i} \chi_{m}\right), H_{m}(\psi) \equiv H_{m n}(\psi), \chi_{m} \equiv$ $\chi_{m n}$. Consider the asymptotics of $G_{1}\left(\vartheta, \Psi, \varphi, \varphi_{0}\right)$ at the limit of large mode numbers $M \gg 1$. We use the formulae

$$
\begin{gathered}
\sum_{n=-\infty}^{\infty} \frac{\mathrm{e}^{\mathrm{i} n \varphi}}{a-n N}=\frac{\pi}{N \sin (\pi a / N)} \mathrm{e}^{\mathrm{i}[\varphi] a / N}, \\
\sum_{n=-\infty}^{\infty} \frac{1}{a-n N}=\frac{\pi}{N \tan (\pi a / N)},
\end{gathered}
$$

where $[\varphi]=\varphi-(2 s+1) \pi / N$ and $2 \pi s / N<\varphi<2 \pi(s+1) / N$, $(s=0, \pm 1, \pm 2, \ldots)$. For the arbitrary time-instants $\varphi, \varphi_{0}$ in the interval $2 \pi s / N<\varphi, \varphi_{0}<2 \pi(s+1) / N$ the generating function $G_{1}\left(\Psi, \vartheta, \varphi, \varphi_{0}\right)$ vanishes for large $M \gg 1$ (supposing that the frequency $\omega(\Psi)$ is finite, $|\omega(\Psi)| \ll M)$ as

$$
G_{1}\left(\vartheta, \Psi, \varphi, \varphi_{0}\right) \sim \mathrm{O}\left(M^{-1}\right) .
$$

However, at the time instants $\varphi=\varphi_{k}=2 \pi s / N \pm 0$ and $\varphi_{0}$ in the interval $2 \pi s / N<\varphi_{0}<2 \pi(s+1) / N$ the generating function $G_{1}\left(\Psi, \vartheta, \varphi, \varphi_{0}\right)$ has a finite asymptotical value for $M \gg 1$, i.e.

$$
\begin{aligned}
& \left.G_{1}\left(\vartheta, \Psi, \varphi, \varphi_{0}\right)\right|_{\varphi=2 \pi s / N \pm 0} \\
& \quad= \pm \frac{\pi}{N} \sum_{m} H_{m}(\Psi) \cos \left(m \vartheta+\chi_{m}\right)+\mathrm{O}\left(M^{-1}\right) .
\end{aligned}
$$

The second-order generating function $G_{2}\left(\vartheta, \Psi, \varphi, \varphi_{0}\right)$ defined according to (35) and (34) as

$$
G_{2}\left(\vartheta, \Psi, \varphi, \varphi_{0}\right)=-\int_{\varphi_{0}}^{\varphi} F_{2}\left(\vartheta\left(\varphi^{\prime}\right), \Psi, \varphi^{\prime}\right) \mathrm{d} \varphi^{\prime}
$$

goes to zero at all $\varphi$ for $M \rightarrow \infty$ because of the definition of the function $F_{2}$ (35) and the asymptotics (39). Similarly, the higher order generating functions $G_{i}\left(\vartheta, \Psi, \varphi, \varphi_{0}\right),(i>2)$ also vanish, because the polynomial functions $F_{i}$ of derivatives $\partial G_{1} / \partial \vartheta, \ldots, \partial G_{i-1} / \partial \vartheta$ on the right-hand side of equations (34) vanish at the limit $M \rightarrow \infty$. Therefore, the generating function $G\left(\vartheta, \Psi, \varphi, \varphi_{0}, \epsilon\right)$ is determined only by the first-order generating function $G_{1}\left(\vartheta, \Psi, \varphi, \varphi_{0}\right)$ for arbitrary values of the perturbation parameter $\epsilon$. The corrections $w_{i}(\Psi, \epsilon)(i \geqslant 1)$ to the perturbed frequency $w(\Psi, \epsilon)(32)$ also vanish at the limit $M \rightarrow \infty$, i.e. $w(\Psi, \epsilon)=\omega(\Psi)$. Thus, the symmetric map (30) can be presented in the form

$$
\begin{gathered}
\Psi_{k}=\psi_{k}-\frac{\epsilon}{2} \frac{\partial S\left(\vartheta_{k}, \Psi_{k}\right)}{\partial \vartheta_{k}}, \\
\Theta_{k}=\vartheta_{k}+\frac{\epsilon}{2} \frac{\partial S\left(\vartheta_{k}, \Psi_{k}\right)}{\partial \Psi_{k}}, \\
\bar{\Theta}_{k}=\Theta_{k}+\frac{2 \pi}{N} \omega\left(\Psi_{k}\right), \\
-\psi_{k+1}=\Psi_{k}-\frac{\epsilon}{2} \frac{\partial S\left(\vartheta_{k+1}, \Psi_{k}\right)}{\partial \vartheta_{k+1}}, \\
\vartheta_{k+1}=\bar{\Theta}_{k}+\frac{\epsilon}{2} \frac{\partial S\left(\vartheta_{k+1}, \Psi_{k}\right)}{\partial \Psi_{k}},
\end{gathered}
$$

where the generating function $S(\vartheta, \Psi)$ is determined by equation (26).

When the perturbation harmonics, $H_{m}(\Psi)$ does not depend on the action variable $\Psi$ the mapping (42) is further simplified. Indeed, in this case $S(\vartheta, \Psi)=S(\vartheta)$ and the map is reduced to

$$
\begin{aligned}
\Psi_{k} & =\psi_{k}-\frac{\epsilon}{2} \frac{\partial S\left(\vartheta_{k}\right)}{\partial \vartheta_{k}}, \\
\vartheta_{k+1} & =\vartheta_{k}+\frac{2 \pi}{N} \omega\left(\Psi_{k}\right), \\
\psi_{k+1} & =\Psi_{k}-\frac{\epsilon}{2} \frac{\partial S\left(\vartheta_{k+1}\right)}{\partial \vartheta_{k+1}} .
\end{aligned}
$$

This map can be called a symmetric radial twist map. The conventional radial twist map (29) can be obtained from (43) for the mapping $\left(\Theta_{k}, \Psi_{k-1}\right) \rightarrow\left(\Theta_{k+1}, \Psi_{k}\right)$ of intermediate variables $(\Theta \equiv \vartheta$, $\Psi)$, i.e.

$\Psi_{k}=\Psi_{k-1}-\epsilon \frac{\partial S\left(\Theta_{k}\right)}{\partial \Theta_{k}}, \quad \Theta_{k+1}=\Theta_{k}+\frac{2 \pi}{N} \omega\left(\Psi_{k}\right)$.

Therefore, the variable $\Psi$ in the radial twist map (44) does not coincide with the corresponding variable of the continuous Hamiltonian system. The map for the original variables $(\vartheta, \psi)$ has a symmetric form (43).

3.4.1. Non-symmetric forms of maps. For the Hamiltonian system (5) and (23) one can also construct maps in the nonsymmetric form (9). However, the generating function of these maps has a more complicated form.

To be more specific, we construct the symplectic map in the form (9). Choosing $\varphi=\varphi_{k}+0=2 \pi s+0$ and $\varphi_{0}=\varphi_{k+1}-0=2 \pi(s+1)-0$ in the generating function (38) and taking the limit $M \rightarrow \infty$ one obtains

$$
\begin{aligned}
S(\vartheta, \psi) & \equiv G_{1}\left(\vartheta, \psi, \varphi_{k}+0, \varphi_{k+1}-0\right) \\
= & \frac{2 \pi}{N} \sum_{m} H_{m}(\psi)\left(-\frac{1}{2} \sin \left[2 \pi m \frac{\omega(\psi)}{N}\right] \sin \left(m \vartheta+\chi_{m}\right)\right. \\
& \left.+\cos ^{2}\left[\pi m \frac{\omega(\psi)}{N}\right] \cos \left(m \vartheta+\chi_{m}\right)\right) .
\end{aligned}
$$

The higher order corrections $G_{i}(i \geqslant 2)$ vanish at the limit $M \rightarrow \infty$. The mapping (30) is then reduced to mapping (9) with the generating function (45).

Therefore, one cannot obtain the perturbed twist map in the form (9), determined by the generating function depending 
only on the perturbation harmonics, $H_{m}(\psi)$, similar to the one in (26). The generating function (45) depends on the variable $\psi$ not only through the radial dependence of the harmonics $H_{m}(\psi)$ but also because of the radial dependence of the frequency $\omega(\psi)$ (or the safety factor $q(\psi)=1 / \omega(\psi)$ ).

The nonsymmetric map of the type (9) for the intermediate variables $(\bar{\Theta}, \Psi)$, i.e. $\left(\bar{\Theta}_{k}, \Psi_{k}\right) \rightarrow\left(\bar{\Theta}_{k+1}, \Psi_{k+1}\right)$, can be approximately obtained from the symmetric map (42) for the small perturbation parameter $\epsilon$. Indeed, using the map (42) the mapping for these variables can be written as

$$
\begin{aligned}
\Psi_{k+1} & =\Psi_{k}-\frac{\epsilon}{2} \frac{\partial}{\partial \vartheta_{k+1}}\left[S\left(\vartheta_{k+1}, \Psi_{k+1}\right)+S\left(\vartheta_{k+1}, \Psi_{k}\right)\right], \\
\bar{\Theta}_{k+1} & =\bar{\Theta}_{k}+\frac{2 \pi}{N} \omega\left(\Psi_{k+1}\right)+\frac{\epsilon}{2}\left[\frac{\partial S\left(\vartheta_{k+1}, \Psi_{k+1}\right)}{\partial \Psi_{k+1}}\right. \\
+ & \left.\frac{\partial S\left(\vartheta_{k+1}, \Psi_{k+1}\right)}{\partial \Psi_{k}}\right] .
\end{aligned}
$$

Using the mapping (42), one can easily show that

$$
S\left(\vartheta_{k+1}, \Psi_{k+1}\right)+S\left(\vartheta_{k+1}, \Psi_{k}\right)=2 S\left(\bar{\Theta}_{k}, \Psi_{k+1}\right)+\mathrm{O}(\epsilon)
$$

and neglecting in equations (46) the terms of order of $\epsilon^{2}$ the mapping can be reduced to the form (9) with the generating function $S\left(\Psi_{k+1}, \bar{\Theta}_{k}\right)$ given by equation (26).

Therefore, the Hamiltonian system (3), (5) and (23) with a broad perturbation spectrum of toroidal modes $n$ can be replaced by the symmetric map (42) with the generating function (26) or by the nonsymmetric map (9) with the generating function given by equation (45). The nonsymmetric map (9) with the simple form (26) of the generating function can be obtained only approximately for the intermediate variables that are not identical to the original variables of the Hamiltonian system, and for the small perturbation parameter $\epsilon$. Then, differences between the original variables and intermediate variables are of the order of the perturbation parameter $\epsilon$.

3.4.2. Standard Hamiltonian and corresponding mappings. In the case $H_{0}(\psi)=\psi^{2} / 2, H_{m}(\psi)=\delta_{m 1}, N=1$ the Hamiltonian (5) and (23) coincides with the standard Hamiltonian. According to (26) the generating function of the standard Hamiltonian is equal to $S(\vartheta)=2 \pi \cos \vartheta$ and the corresponding map takes the form

$$
\begin{gathered}
\Psi_{k}=\psi_{k}+\frac{\epsilon}{2} \sin \vartheta_{k}, \\
\vartheta_{k+1}=\vartheta_{k}+2 \pi \Psi_{k}, \\
\psi_{k+1}=\Psi_{k}+\frac{\epsilon}{2} \sin \vartheta_{k+1} .
\end{gathered}
$$

This map, called the symmetric standard map, was first obtained in [39] for small values of $\epsilon$, and for arbitrary values of $\epsilon$ in [40].

The traditional forms of the standard map may be obtained from the symmetric radial map (47) for the variables $\left(\Psi_{k}, \vartheta_{k}\right)$, i.e.

$$
\Psi_{k}=\Psi_{k-1}+\epsilon \sin \vartheta_{k}, \quad \vartheta_{k+1}=\vartheta_{k}+2 \pi \Psi_{k}
$$

for the mapping $\left(\vartheta_{k}, \Psi_{k-1}\right) \rightarrow\left(\vartheta_{k+1}, \Psi_{k}\right)$ or

$$
\vartheta_{k+1}=\vartheta_{k}+2 \pi \Psi_{k}, \quad \Psi_{k+1}=\Psi_{k}+\epsilon \sin \vartheta_{k+1}
$$

for the mapping $\left(\vartheta_{k}, \Psi_{k}\right) \rightarrow\left(\vartheta_{k+1}, \Psi_{k+1}\right)$. One can see from these mappings that the action variable $\Psi$ in the standard map does not coincide with the action variable $\psi$ in the Hamiltonian (5) and (23).

Phase portraits of the symmetric standard map (47), the standard maps (48) and its nonsymmetric form (9) with the generating function (45) are shown in figure 2 for the perturbation parameter $\epsilon=0.7 / 2 \pi$. The symmetric standard map (47) reproduces very well the Poincaré section obtained by the integration of the Hamiltonian system (5) and (23) using the symmetric map (30) with the generating function (38) with a finite number $M$ (see [40]). The map (9) with the generating function (45) (see figure 2(c)) also reproduces this result but with less accuracy.

\subsection{Numerical mapping methods}

Besides analytical mapping models there also exist several numerical mapping techniques for integrating Hamiltonian systems, particularly, the equations of magnetic field lines and particles in toroidal plasmas. These mappings have been employed for modelling heat and particle transport in toroidal stochastic plasmas (see, e.g. [68-73]). We briefly describe below the main idea of the method known as Interpolated Cell Mapping. The method uses real space coordinates $\boldsymbol{x}=(x, y, \varphi)$, which may be cylindrical coordinates $(R, Z, \varphi)$, where $R$ is a major radius and $Z$ is a vertical coordinate, or toroidal coordinates $(r, \theta, \varphi)$. One introduces $N_{\varphi}$ cross sections $\varphi_{k}$ equally spaced along the toroidal angle $\varphi$. The relation

$\left(x_{k+1}, y_{k+1}\right)=\hat{M}\left(x_{k}, y_{k}\right)=\left(M_{x}^{k}\left(x_{k}, y_{k}\right), M_{y}^{k}\left(x_{k}, y_{k}\right)\right)$,

defines the map of field line coordinates $\left(x_{k}, y_{k}\right)$ at the sections $\varphi_{k}$ to the ones $\left(x_{k+1}, y_{k+1}\right)$ at $\varphi_{k+1}$, determined by the set of functions $M_{x}^{k}(x, y), M_{y}^{k}(x, y), k=1,2, \ldots, N_{\varphi}$.

In order to create these functions the field line equations (1) are integrated numerically from grid points $\left(x_{k, i j}, y_{k, i j}\right)$ at the section $\varphi_{k}$ to the intersection point $\left(M_{x}^{k}\left(x_{k, i j}, y_{k, i j}\right), M_{y}^{k}\left(x_{k, i j}, y_{k, i j}\right)\right)$ at the next section $\varphi_{k+1}$. The functions $M_{x}^{k}(x, y), M_{y}^{k}(x, y)$ are interpolated by the cubic splines from their computed values at the grid points $\left(x_{k, i j}, y_{k, i j}\right)$. Once the functions $M_{x}^{k}(x, y), M_{y}^{k}(x, y), k=$ $1,2, \ldots, N_{\varphi}$ have been created one can follow field lines using the map (50), which run much faster than direct numerical integration of the equations of field lines. This is a main advantage of this numerical mapping technique. A similar numerical mapping technique called the 'Reversible Field-Line Mapping Technique' has been proposed in [73].

One of the main shortcomings of these methods is that the numerical mapping (50) is not symplectic. Since the mapping variables $(x, y)$ are not canonical variables it is hard to impose conditions on the functions $M_{x}^{k}(x, y), M_{y}^{k}(x, y)$ that would make mappings symplectic (or flux-preserving).

\section{The symmetric tokamap}

In this section, we discuss the relation of the tokamap with the corresponding continuous Hamiltonian system and using the results of the previous section we propose the symmetric form of the tokamap, which describes the continuous system more accurately than the tokamap. 
4.1. The continuous Hamiltonian system and the symmetric tokamap

One should note that the derivation of the tokamap from a continuous Hamiltonian system is not a well-defined problem unless we make some additional assumptions. The main difficulty lies in determining to which kind of a continuous Hamiltonian system the tokamap corresponds. In order to make this problem unambiguous we suppose that the perturbation Hamiltonian $H_{1}(\psi, \vartheta, \varphi)$ as well as the generating function $S(\vartheta, \psi)(17)$ of the tokamap do not depend on the safety factor $q(\psi)$.

In general, as was shown in section 3.4 symplectic mappings of type (9) with the simple generating function $S$ (26) like the one of the tokamap (17) cannot be constructed for Hamiltonian systems of type (5) with radially dependent perturbation functions $H_{1}(\psi, \vartheta, \varphi)$. It means that the tokamap cannot be rigorously derived from the Hamiltonian field line equation (5) under the constraint on the Hamiltonian and generating function imposed above in order to have a simple mapping model. We study this problem by constructing a mapping for the following continuous Hamiltonian system corresponding to the tokamap

$$
\begin{gathered}
H=\int \frac{\mathrm{d} \psi}{q(\psi)}+\epsilon H_{1}(\psi) \sum_{n=-M}^{M} \cos (\vartheta-n \varphi), \\
\epsilon H_{1}(\psi)=-\frac{\epsilon}{2 \pi} \frac{\psi}{1+\psi},
\end{gathered}
$$

with a large number, $2 M+1 \gg 1$, of toroidal modes $n$. In the limit $M \rightarrow \infty$ the Hamiltonian (51) is reduced to

$H=\int \frac{\mathrm{d} \psi}{q(\psi)}+\epsilon 2 \pi H_{1}(\psi) \cos \vartheta \sum_{k=-\infty}^{\infty} \delta(\varphi-2 \pi k)$,

which has been used in [63] to analyse its relation with the tokamap. The integration of field line equation (25) corresponding to the Hamiltonian (52) would give the tokamap (16) if the integrals (27) containing delta functions are replaced by $f\left(\vartheta_{k}, \psi_{k+1}\right)$ (see section 3.1). As was noted above, this procedure is not justified when the perturbation Hamiltonian $H_{1}$ is dependent on the toroidal flux $\psi$.

The continuous Hamiltonian (52) is a particular case of the Hamiltonian (23). According to results of section 3.4 only the symmetric form (42) of the map (7) corresponding to the system (23) or (52) has the simple generating function (26). The generating function (45) of the nonsymmetric mappings (9) has a rather complicated dependence on the perturbation harmonics $H_{m}(\psi)$

According to equations (42) the symmetric map corresponding the tokamap Hamiltonian (52) can be written as

$$
\begin{array}{r}
\Psi_{k}=\psi_{k}-\frac{\epsilon}{2} \frac{\Psi_{k}}{1+\Psi_{k}} \sin \vartheta_{k}, \\
\Theta_{k}=\vartheta_{k}-\frac{\epsilon}{2} \frac{1}{\left(1+\Psi_{k}\right)^{2}} \cos \vartheta_{k}, \\
\bar{\Theta}_{k}=\Theta_{k}+\frac{2 \pi}{q\left(\Psi_{k}\right)}, \\
\psi_{k+1}=\Psi_{k}-\frac{\epsilon}{2} \frac{\Psi_{k}}{1+\Psi_{k}} \sin \vartheta_{k+1}, \\
\vartheta_{k+1}=\bar{\Theta}_{k}-\frac{\epsilon}{2} \frac{1}{\left(1+\Psi_{k}\right)^{2}} \cos \vartheta_{k+1},
\end{array}
$$

which we call the symmetric tokamap. The generating function $S(\vartheta, \psi)$ of this map is the same as that of the tokamap (17).

In general, the symmetric tokamap is an implicit map unlike the tokamap. The first equation in (53) can be explicitly resolved with respect to $\Psi_{k}$, similarly to the tokamap,

$$
\begin{gathered}
\Psi_{k}=\frac{1}{2}\left[\sqrt{P^{2}\left(\psi_{k}, \vartheta_{k}\right)+4 \psi_{k}}-P\left(\psi_{k}, \vartheta_{k}\right)\right], \\
P\left(\psi_{k}, \vartheta_{k}\right)=1-\psi_{k}+\frac{\epsilon}{2} \sin \vartheta_{k} .
\end{gathered}
$$

However, the last equation in the mapping (53) cannot be explicitly written with respect to the variable $\vartheta_{k+1}$. The latter should be found numerically using, for instance, the Newton method. According to section 3.4.1, the tokamap (2.2.3) can be obtained from the symmetric tokamap (53) for the intermediate variables $(\bar{\Theta}, \Psi)$, at small perturbations $\epsilon \ll 1$.

\subsection{Comparison of the tokamap and the symmetric tokamap}

The symmetric tokamap is invariant with respect to the translation $\vartheta \leftrightarrow \pi-\vartheta$. It reflects the invariance of the symmetric map with respect to the transformation $k \leftrightarrow k+1$ with the simultaneous change $K \rightarrow-K$ and $q \rightarrow-q$. The property corresponds to the symmetry of the continuous Hamiltonian system with respect to the formal transformation $t \rightarrow-t, H \rightarrow-H$.

Fixed points $\left(\vartheta_{k}, \psi_{k}\right)$ of the tokamap and the symmetric tokamap, defined by $\psi_{k+1}=\psi_{k}, \vartheta_{k+1}=\vartheta_{k}+2 \pi n$ where $n$ is an integer number, are the same. They either lie on the polar axis $\psi_{k}=0$, or on the equatorial plane $\vartheta_{k}=0, \pi, \sin \vartheta_{k}=0$. For the last case, $\psi_{k}$ are roots of the equation ( [11])

$$
\frac{1}{q(\psi)} \mp \frac{\epsilon}{2 \pi} \frac{1}{(1+\psi)^{2}}-n=0,
$$

where the signs (干) corresponds to $\vartheta_{k}=0$ and $\vartheta_{k}=\pi$, respectively.

However, the periodic fixed points, $(\vartheta, \psi)=\hat{M}^{q}(\vartheta, \psi)$, $(q>1)$, of the tokamap and the symmetric tokamap are different. They have been studied for the tokamap case in [61]. Since the tokamap cannot be exactly obtained from the symmetric map, the fixed points of the symmetric tokamap cannot be recovered from those of the tokamap. The deviation of these maps depends significantly on the perturbation parameter $\epsilon$. It is of the order of $\epsilon$. For small values, $\epsilon \ll 1$ it is small; however, for $\epsilon \sim 1$ the difference becomes relatively large. We will study below the difference between the symmetric tokamap and the tokamap by comparing their phase portraits. A more detailed study of fixed points of the symmetric map requires a separate investigation and we will not discuss this problem here.

Figure 4 shows phase portraits of the tokamap (16) and the symmetric tokamap (53) (red dots) for the profile of the safety factor, (18), at the perturbation parameter $\epsilon=2.55 / 2 \pi$ : (a) and $(c)$ corresponds to the tokamap, and $(b)$ and $(d)$ to the symmetric tokamap. Figures $4(a)$ and $(b)$ describe the phase portrait on the $(\vartheta, \psi)$-plane, while $4(c)$ and $(d)$ corresponds to that on the polar plane $(\psi \cos \vartheta, \psi \sin \vartheta)$.

For comparison we have also integrated the continuous Hamiltonian (51) with a finite number of toroidal modes $M=5$ using the mapping (30). They are plotted in 

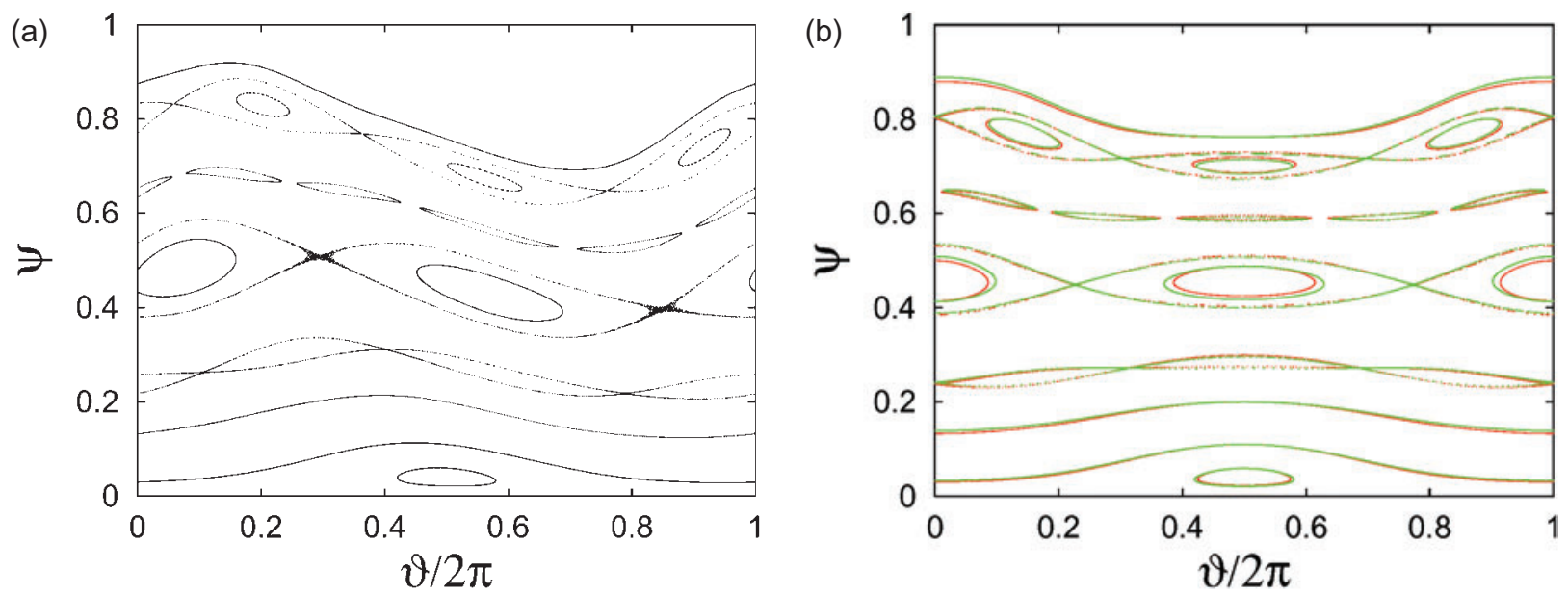

(c)

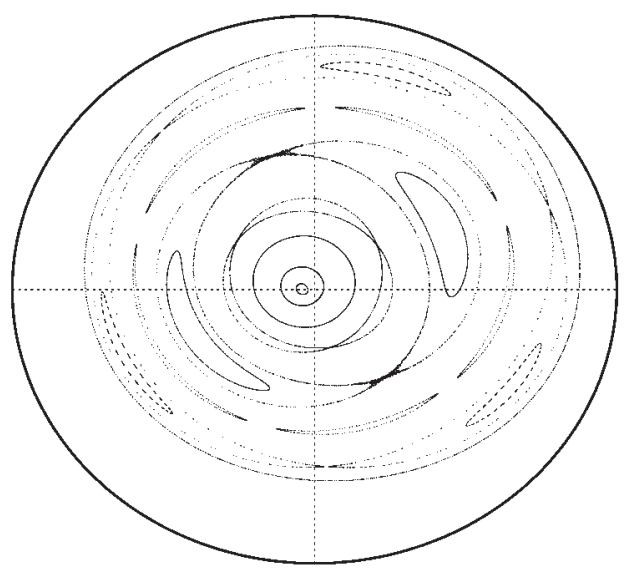

(d)

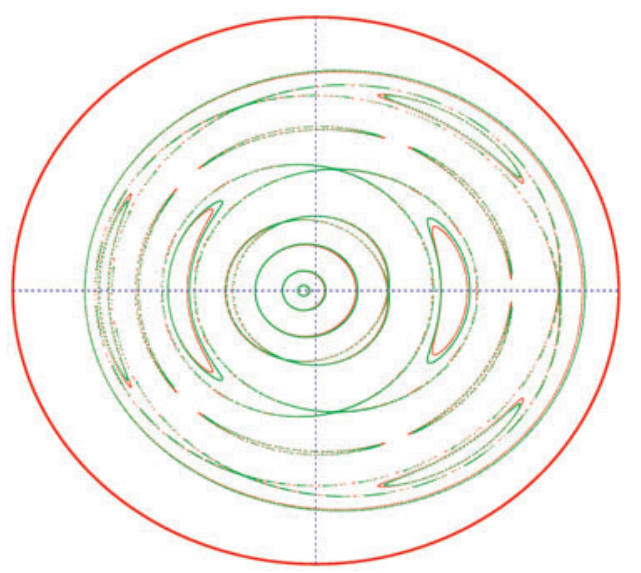

Figure 4. Phase portraits of the tokamap (16) $(a)$ and $(c)$, and the symmetric tokamap (red dots) and the symmetric map (30) (green dots) $(b)$ and $(d)$ with the Hamiltonian (51). The $q$-profile is given by (18); parameters $\epsilon=2.55 / 2 \pi, q(0)=1, M=5$.

figures $4(b)$ and $(d)$ by green dots. The toroidal angle step for the map is taken to be $\Delta \varphi=2 \pi$.

As seen from the figures the symmetric tokamap very closely describes the continuous Hamiltonian system (51) with a finite number of toroidal modes $M$. However, the phase curves of the tokamap are more distorted than in the symmetric tokamap. The positions of the periodic fixed points are shifted not only radially, but also along the poloidal angle $\vartheta$. The difference between the tokamap and the symmetric tokamap becomes more pronounced for large perturbation parameters, $\epsilon$.

To illustrate this we compare the perturbation thresholds $\epsilon_{g}$ of destruction of the so-called golden KAM curve corresponding to the tokamap and the symmetric tokamap. For the tokamap this problem was discussed in [11]. The golden KAM curve is the most robust KAM barrier in the standard map, and it corresponds to the winding number $\omega$ equal to $g_{*}=G_{*}^{-1}=0.6180339 \ldots$, where $G_{*}$ is the golden section defined by the equation: $G_{*}^{2}=G_{*}+1$. For the safety factor $q(\psi)=1 / \omega(\psi)(18)$ the golden KAM curve is located at $\psi_{g}=0.31599$. It lies between two periodic orbits $(m, n)\left(q\left(\psi_{m n}\right)=m / n\right)$ corresponding to $q\left(\psi_{2,1}\right)=2: 1$ located at $\psi_{2,1} \approx 0.45631$ and $q\left(\psi_{4,3}\right)=4: 3$ located at $\psi_{4,3} \approx 0.18946$. For the finite perturbation parameter $K>0$ the KAM curves are deformed or broken. The periodic orbits are replaced by islands. With increasing $K$ the width of the islands grows destroying the KAM curves located between two neighbouring islands. According to [11] the golden KAM curve $\psi_{g}$ corresponding to the tokamap is destroyed at $\epsilon_{g}=4.3 / 2 \pi$. However, at this value of $\epsilon$, the golden KAM curve of the symmetric tokamap survives. This is shown in figure 5 for the perturbation parameter $\epsilon=4.5 / 2 \pi$ : (a) the tokamap; (b) the symmetric tokamap.

In the tokamap case several islands including $2: 1$ and $4: 3$ are already overlapped forming a single stochastic belt. But in the case of the symmetric tokamap most of these islands are still isolated. From figure $5(b)$ one can see a gap between the islands $2: 1$ and $4: 3$. The golden KAM curve of the symmetric tokamap is destroyed at larger values of $\epsilon$ equal to $\epsilon_{g} \approx 4.8 / 2 \pi$. The phase portrait of the symmetric tokamap at $\epsilon=5 / 2 \pi$ is shown in figure 6 .

\subsection{Revtokamap and the symmetric revtokamap}

Consider another example illustrating a difference between the tokamap (or revtokamap) and the symmetric tokamap (revtokamap) in the case of a reversed magnetic shear configuration described by the safety factor (19) (see also figure $3(b)$ ). The mappings in this case are non-twist maps. The dynamics of the revtokamap in such a reversed magnetic shear has been studied in [12] in great detail. Here, we will only compare the symmetric revtokamap and the revtokamap 

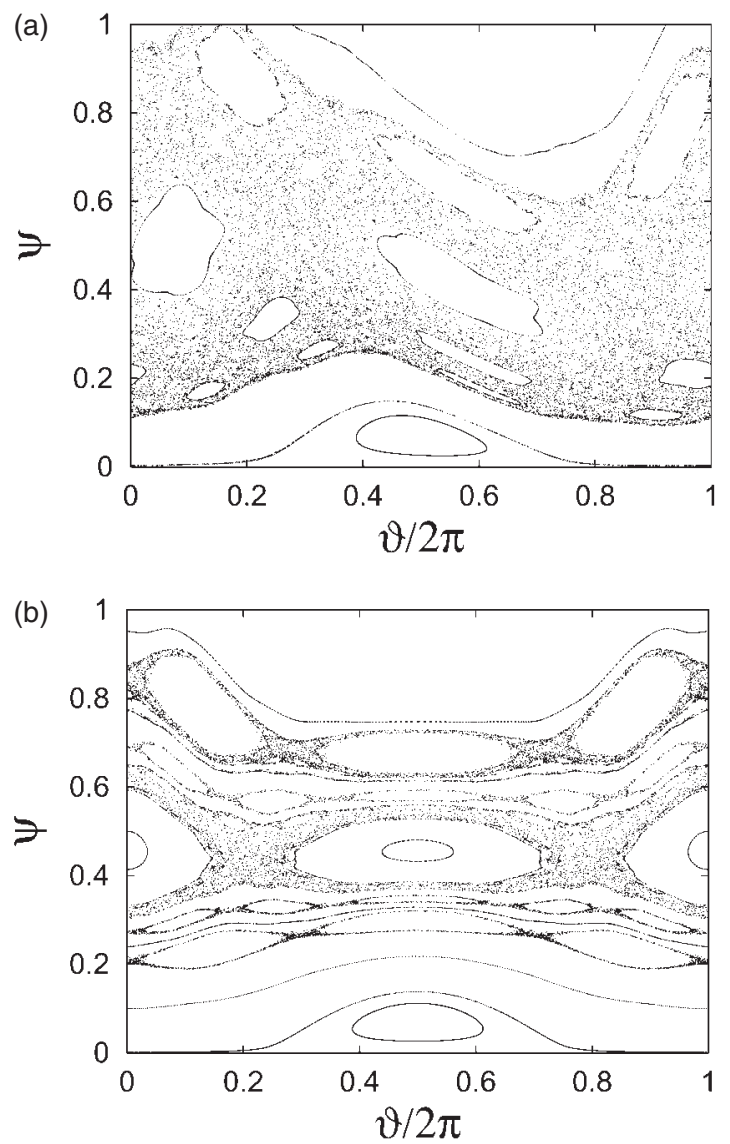

Figure 5. Stochastic belt of the tokamap (16) (a) and the symmetric tokamap $(b)$ with the $q$-profile (18) for $K=4.5 / 2 \pi, q(0)=1$.

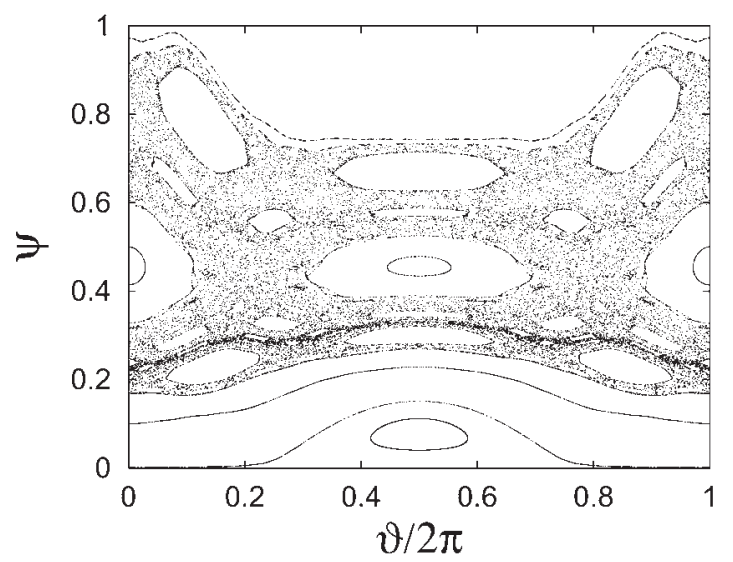

Figure 6. The same as in figure $5(b)$ but for $\epsilon=2.5 / \pi, q(0)=1$.

at large values of the perturbation parameter $\epsilon$ when the difference between these maps is expected to be large.

Phase portraits of the maps are plotted in figure 7 for the perturbation parameter $\epsilon=6.3 / 2 \pi:(a)$ the revtokamap; (b) the symmetric tokamap. The values of the safety factor $q(\psi)$ are chosen to be equal to $q_{0}=3$ at the magnetic axis, $q_{m}=1.5$ at the minimum, and $q_{1}=6$ at the edge, as in [12]. Then, the position of the shearless curve is at $\psi_{m}=0.44948974$, where $q^{\prime}\left(\psi_{m}\right)=0$.

The most robust invariant curves are located near the shearless curve $\psi=\psi_{m}$. According to the revtokamap the
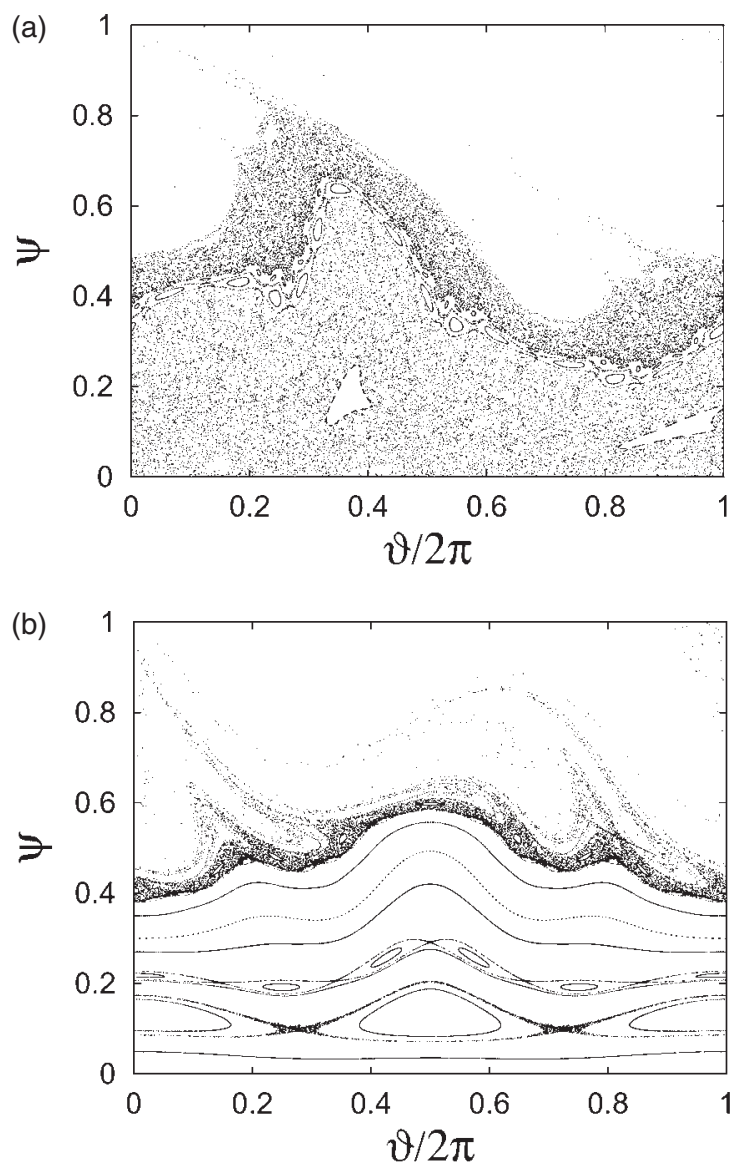

Figure 7. Phase portraits of the tokamap (a) and the symmetric tokamap $(b)$ with the reversed $q$-profile (19); parameters $\epsilon=6.3 / 2 \pi, q_{0}=3, q_{m}=1.5$ and $q_{1}=6$.

upper region $\psi>\psi_{m}$ and the lower region $\psi<\psi_{m}$ of field lines develop into the chaotic belts: the upper region $\psi>\psi_{m}$ is open, while the lower region is confined. These regions are separated from each other by the transport barrier located near the perturbed shearless curve (see figure 7(a)). However, the symmetric revtokamap with the same perturbation parameter $\epsilon$ gives a rather different picture. Although, the upper region $\psi>\psi_{m}$ of open field lines is completely chaotic, the lower region $\psi<\psi_{m}$ consists of almost regular field lines with a few isolated islands (see figure $7(b)$ ). This result can also be confirmed by direct integration of the continuous Hamiltonian system (51). In general, the tokamap, systematically, gives lower critical values of the perturbation parameter, $\epsilon_{g}$, of destruction of the KAM curves than the symmetric tokamap.

\section{Model of magnetic field in the presence of external perturbations}

In this section, we consider a model describing magnetic field lines in the plasma affected by external magnetic perturbations. The model is given by the Hamiltonians

$H=\int \frac{\mathrm{d} \psi}{q(\psi)}+\epsilon \sum_{m} H_{m}(\psi) \cos (m \vartheta-n \varphi)$,

$H_{m}(\psi)=(-1)^{m} \frac{\sin \left(m-m_{0}\right) \vartheta_{d}}{\pi\left(m-m_{0}\right)} \psi^{m / 2}$. 


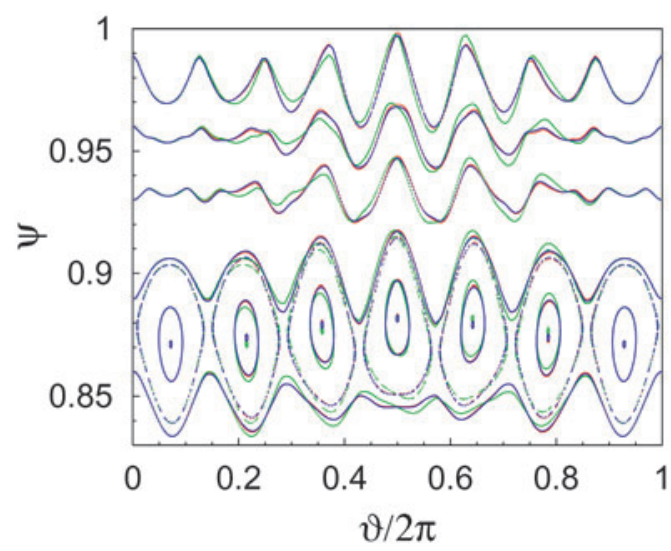

Figure 8. Phase portraits of the model (54) obtained by the symplectic mapping integration (30) (red dots), using the symmetric map (42) (blue dots) and nonsymmetric map (9) (green dots). The perturbation parameter $\epsilon=5 \times 10^{-4}$, the $q$-profile is given by (18) with $q_{0}=1$.

It presents the main features of magnetic field lines in a so-called ergodic divertor tokamak $[14,16]$. The perturbation is localized in a finite poloidal angle region, $\pi-\vartheta_{d}<$ $\vartheta<\pi+\vartheta_{d}$, with the extension $\Delta \vartheta=2 \vartheta_{d}$. The poloidal mode spectrum $H_{m}(\psi)$ is centred near the central mode $m_{0}$ with width $\Delta m=\pi / \vartheta_{d}$. Furthermore, we will use the safety factor (18) (see also figure $3(a)$ ), and choose $m_{0}=6$ and $n=2$ so that the rational magnetic surface $\psi_{m n}$, $q\left(\psi_{m n}\right)=m_{0} / n=3$, corresponding to this mode is located near $\psi_{m n} \approx 0.8$. The parameter $\vartheta_{d}$ is equal to $\pi / 3$, and the sum over $m$ in (54) is taken from $m_{0}-4$ to $m_{0}+4$.

We have modelled the Hamiltonian system (54) by the symmetric and nonsymmetric mappings given by equations (42) and (9), respectively, with the same generating function (26). These mapping models are compared with the direct integration of the Hamiltonian system (54) using the symplectic mapping (30). The integration step is taken to be equal to $\Delta \varphi=\pi / 8$. The corresponding phase portraits at the peripheral region of the plasma where the effect of perturbation is large are shown in figure 8: red dots describe direct integration, blue dots correspond to the symmetric mapping model (42), and green dots to the nonsymmetric mapping model (9). The perturbation parameter is $\epsilon=$ $5 \times 10^{-4}$, the $q$-profile is given by (18) with $q_{0}=1$. As seen from figure 8 the symmetric mapping model describes the system much better than the nonsymmetric mapping model, even for such a small value of perturbation. The symmetric map describes the positions of periodic fixed points better than the nonsymmetric one.

With increasing perturbation $\epsilon$ several $m: n$ resonances located at the plasma periphery start to overlap forming a stochastic layer. Field lines leave the stochastic layer when they cross the plasma boundary $\psi=1$. We have calculated a probability $f(\varphi)$ of field lines to remain in the plasma after rotation $\varphi$ along the toroidal angle. In order to determine this quantity, a large number, $N_{0}=6 \times 10^{4}$, of field lines with coordinates inside the stochastic layer are launched. Then, the probability $f(\varphi)$ is determined as a ratio of the number of orbits $N(\varphi)$ that did not leave the plasma after rotation $\varphi$ to the full number of orbits $N_{0}$, i.e. $f(\varphi)=N(\varphi) / N_{0}$. The dependence

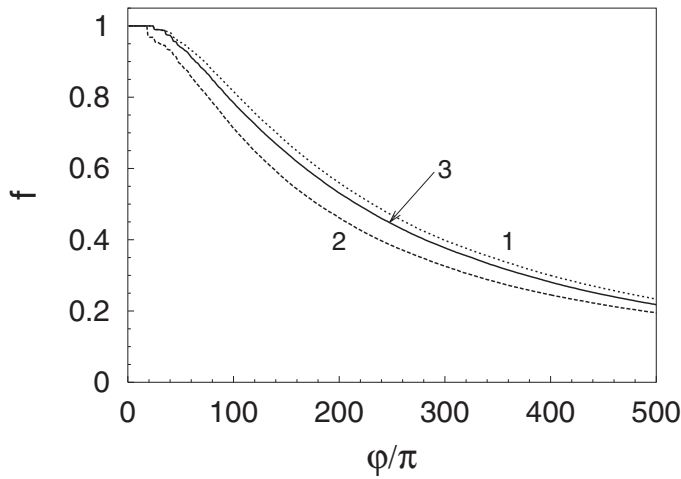

Figure 9. Fraction of orbits, $f(\varphi)$, which did not leave the plasma after rotation $\varphi$ along the toroidal direction calculated by three different methods: dotted curve 1 corresponds to the symmetric map (42); dashed curve 2 to the nonsymmetric map (9); solid curve 3 to the symplectic mapping integration (30). The perturbation parameter $\epsilon=4 \times 10^{-3}$, the $q$-profile is given by (18) with $q_{0}=1$.

of $f(\varphi)$ on the angle $\varphi$ obtained by three different methods is shown in figure 9: dotted curve 1 corresponds to the symmetric map (42); dashed curve 2 to the nonsymmetric map (9); solid curve 3 to the symplectic mapping integration (30). The perturbation parameter $\epsilon=4 \times 10^{-3}$. Figure 9 shows that field lines remain in the stochastic layer for some transient 'time' $\varphi_{0}$ before any field line leaves it. It is seen that the symmetric mapping model describes the behaviour of $f(\varphi)$, especially the transient time $\varphi_{0}$, better than the nonsymmetric mapping model. The symmetric mapping model yields $\varphi_{0}=25 \pi$ coinciding with the result of the symplectic mapping integration while the nonsymmetric map gives a smaller value $\varphi_{0}=19 \pi$. As in the case of the tokamap, the nonsymmetric map model has lower threshold values of $\varphi_{0}$ than the symmetric mapping model.

\section{Conclusions}

In conclusion, we would like to make general remarks on mapping models of magnetic field lines in toroidal plasmas in the presence of magnetic perturbations. Usually, these perturbations depend on the radial coordinate, which is manifest in the dependence of the perturbation Hamiltonian $H_{1}$ (6) on the toroidal flux $\psi$. In general studies of field lines one wishes to replace the continuous equations of magnetic field lines in toroidal plasmas with the simple discrete model mapping of field lines. These model mappings should satisfy several requirements or constraints that would make them compatible with the properties of Hamiltonian equations of field lines and the toroidal geometry of plasmas. An example of such requirements was given in section 2.2.3. Besides the mappings being symplectic and compatible with the toroidal geometry, one wishes to construct a mapping whose generating function would have a simple dependence on the radially dependent perturbation field. In section 3.4, we have shown that only the symmetric symplectic map of type (42) derived from the Hamiltonian equations satisfies this constraint. The nonsymmetric form of the mapping (9) which can also be derived from a Hamiltonian system has a complicated dependence on the perturbation field $H_{1}$ and the 
frequency of motion $\omega(\psi)$ (or the safety factor $q(\psi)$ ) (see the generating function (45)).

Therefore, when one wishes to construct a model map for magnetic field lines in toroidal plasmas, one should impose an additional constraint along with others mentioned in section 2.2.3, namely the map should be constructed in a symmetric form of type (42) with the generating function $S(\vartheta, \Psi)$ in the form (26). The form of perturbation functions $H_{m}(\Psi)$ can be established by other constraints listed in section 2.2.3. Such mapping models would be more compatible with Hamiltonian equations of field lines, and they describe original Hamiltonian systems better.

Symmetric mapping models of Hamiltonian systems proposed in this work should also be useful in some important problems of accelerator physics and dynamical astronomy. The symplectic mapping models have been important tools to study the long-term stability of particle motion in accelerators (see [29-32]). In most cases, they have been constructed in a nonsymmetric form, which is less compatible with the original Hamiltonian system. Similar mapping models have been used in dynamical astronomy to study the long-term behaviour of small planetary objects, asteroids and comets, in the Solar System [33-38]. The use of symmetric mapping models would significantly increase the accuracy of these studies.

\section{Acknowledgments}

The author would like to thank Professor G. Eilenberger, Dr T. Evans and Professor D. Reiter for fruitful discussions and comments. He is also grateful to referees for valuable comments and suggestions. This work has been done in the frame of the project SFB 591 of the Deutsche Forschungsgemeinschaft (DFG).

\section{References}

[1] Poincaré H. 1992 New Methods of Celestial Mechanics vol 1-3 (New York: AIP)

[2] Boozer A.H. 1983 Phys. Fluids 261288

[3] Boozer A.H. 1992 Plasma confinement Encyclopedia of Physical Science and Technology vol 13 (New York: Academic)

[4] Morrison P.J. 2000 Phys. Plasmas 72279

[5] Arnold V.I. 1989 Mathematical Methods of Classical Mechanics (Berlin: Springer)

[6] Wobig H. 1987 Z. Naturf. a 421054

[7] Wobig H. and Fowler R.H. 1988 Plasma Phys. Control. Fusion 30721

[8] Bazzani A., Malavasi M. and Siboni M. 1989 Il Nuovo Cimento 103659

[9] Mendonça J.T. 1991 Phys. Fluids B 387

[10] Bazzani A., Di Sebastiano A. and Turchetti G. 1998 Il Nuovo Cimento D 201795

[11] Balescu R., Vlad M. and Spineanu F. 1998 Phys. Rev. E 58951

[12] Balescu R. 1998 Phys. Rev. E 583781

[13] Martin T.J. and Taylor J.B. 1984 Plasma Phys. Control. Fusion 26321

[14] Abdullaev S.S., Finken K.H., Kaleck A. and Spatschek K.H. 1998 Phys. Plasmas 5196

[15] Fischer O. and Cooper W.A. 1998 Plasma Phys. Rep. 24727

[16] Abdullaev S.S., Finken K.H. and Spatschek K.H. 1999 Phys. Plasmas 6153

[17] Da Silva E.C., Caldas I.L. and Viana R.L. 2001 Phys. Plasmas 82855
[18] Da Silva E.C., Caldas I.L. and Viana R.L. 2001 IEEE Trans. Plasma Sci. 29617

[19] da Silva E.C., Caldas I.L., Viana R.L. and Sanjuán M.A.F. 2002 Phys. Plasmas 94917

[20] Punjabi A., Verma A. and Boozer A.H. 1992 Phys. Rev. Lett. 693322

[21] Punjabi A., Verma A. and Boozer A.H. 1994 J. Plasma Phys. 5291

[22] Punjabi A., Verma A. and Boozer A.H. 1996 J. Plasma Phys. 56569

[23] Punjabi A., Ali H. and Boozer A.H. 1997 Phys. Plasmas 4337

[24] Yamagishi T. 1995 Fusion Technol. 27505

[25] Abdullaev S.S. and Zaslavsky G.M. 1995 Phys. Plasmas 2 4533

[26] Abdullaev S.S. and Zaslavsky G.M. 1996 Phys. Plasmas 3516

[27] Abdullaev S.S. and Finken K.H. 1998 Nucl. Fusion 38531

[28] Punjabi A., Ali H. and Boozer A.H. 2003 Phys. Plasmas 10 3992

[29] Bazzani A., Todesco E., Turchetti G. and Servizi G. 1994 A normal form approach to the theory of nonlinear betatronic motion CERN Report 1994 No 94-02, p 225

[30] Berg S.J., Warnock R.L., Ruth R.D. and Forest É. 1994 Phys. Rev. E 49722

[31] Dragt A.J. 1996 Particle Accelerators 55253

[32] Warnock R.L. and Berg S.J. 1997 AIP Conf. Proc. vol 395 (Woodbury, NY: American Institute of Physics) pp 423-45

[33] Wisdom J. 1982 Astron. J. 87577

[34] Wisdom J. and Holman M. 1991 Astron. J. 1021528

[35] Wisdom J., Holman M. and Touma J. 1996 Integration Algorithms for Classical Mechanics vol 10, ed J.E. Marsden et al (Rhode Island: Field Institute Communications) pp 217-44

[36] Hadjidemetriou J.D. 1991 Predictability, Stability and Chaos in N-body Dynamical Systems ed A. Roy (Dordrecht: Kluwer) p 157

[37] Hadjidemetriou J.D. 1998 Analysis and Modelling of Discrete Dynamical Systems ed C. Froeschle and D. Benest (London: Gordon and Breach) chapter 9, pp 249-82

[38] Šidlichovský M. 1997 Celes. Mech. Dynam. Astron. 6569

[39] Abdullaev S.S. 1999 J. Phys. A: Math. Gen. 322745

[40] Abdullaev S.S. 2002 J. Phys. A: Math. Gen. 352811

[41] Balescu R. 1988 Transport Processes in Plasmas (Amsterdam: North-Holland)

[42] Sanz-Serna J.M. 1991 Acta Numer. 243

[43] Sanz-Serna J.M. and Calvo M.P. 1994 Numerical Hamiltonian problems Appl. Math. Math. Comput. vol 7 (London: Chapman and Hall)

[44] Chirikov B.V. 1979 Phys. Rep. 52265

[45] Rechester A.B., Rosenbluth M.N. and White R.B. 1979 Phys. Rev. Lett. 421247

[46] Rechester A.B. and White R.B. 1980 Phys. Rev. Lett. 441586

[47] Rechester A.B., Rosenbluth M.N. and White R.B. 1981 Phys. Rev. A 232664

[48] Ichikawa Y.H., Kamimura T. and Hirose K. 1987 Physica D 29247

[49] Rax J.M. and White R.B. 1992 Phys. Rev. Lett. 681523

[50] Horton W., Park H.-B., Kwon J.-M., Strozzi D., Morrison P.J. and Choi D.-I. 1998 Phys. Plasmas 53910

[51] Kwon J.-M., Horton W., Zhu P., Morrison P.J., Park H.-B. and Choi D.-I. 2000 Phys. Plasmas 71169

[52] Tabet R., Saifaoui D., Dezairi D. and Raouak A. 1998 Eur. Phys. J.: Appl. Phys. 4329

[53] Tabet R., Imrane H., Saifaoui D., Dezairi D. and Miskane F. 2000 Eur. Phys. J.: Appl. Phys. 12145

[54] Miskane F., Dezairi D., Saifaoui D., Imzi H., Imrane H. and Benharraf M. 2001 Eur. Phys. J.: Appl. Phys. 13205

[55] del-Castillo-Negrete D. and Morrison P.J. 1993 Phys. Fluids A 5948

[56] del-Castillo-Negrete D., Greene J.M. and Morrison P.J. 1996 Physica D 911

[57] Litaudon X. 1998 Plasma Phys. Control. Fusion 40 A251

[58] Wolf R. 2003 Plasma Phys. Control. Fusion 45 R1 
[59] Misguich J.H. 2001 Phys. Plasmas 82132

[60] Misguich J.H., Reuss J.D., Consrtantinescu D., Steinbrecher G., Vlad M., Spineanu F., Weyssow B. and Balescu R. 2002 Noble internal transport barriers and radial subdiffusion of toroidal magnetic lines (CEA/Cadarache, 2002) Preprint EUR-CEA-FC-1724

[61] Misguich J.H., Reuss J.D., Consrtantinescu D.,

Steinbrecher G., Vlad M., Spineanu F., Weyssow B. and Balescu R. 2002 Plasma Phys. Control. Fusion 44 L29-35

[62] Weyssow B. and Misguich J.H. 1999 Europhys. Conf. Abstracts J 23793

[63] Eberhard M. 1999 Europhys. Conf. Abstracts J 23781

[64] Lichtenberg A.J. and Lieberman M.A. 1992 Regular and Stochastic Motion 2nd edn (New York: Springer)

[65] Wobig H. and Pfirsch D. 2001 Plasma Phys. Control. Fusion 43695
[66] Zaslavsky G.M. 1985 Stochasticity of Dynamical Systems (Chur, Switzerland: Harwood Academics)

[67] Zaslavsky G.M., Sagdeev R.Z., Usikov D.A. and Chernikov A.A. 1991 Weak Chaos and Quasi-Regular Patterns (Cambridge: Cambridge University Press)

[68] Tongue B.H. 1987 Physica D 8401

[69] Montvai A. and Düchs D.F. 1993 Physics Computing'92 (Prague, 1992) (Singapore: World Scientific) p 417

[70] de Rover M., Lopes Cardozo N.J. and Montvai A. 1996 Phys. Plasmas 34478

[71] Kasilov S.V., Moiseenko V.E. and Heyn M.F. 1997 Phys. Plasmas 42422

[72] Runov A.M., Reiter D., Kasilov S.V., Heyn M.F. and Kernbichler W. 2001 Phys. Plasmas 82422

[73] Feng Y., Sardei F., Grigull P., McCormick K., Kisslinger J., Reiter D. and Igitkhanov Y. 2002 Plasma Phys. Control. Fusion 44611 